\title{
- Glycan processing in the Golgi : \\ = optimal information coding and \\ . constraints on cisternal number and \\ . enzyme specificity
}

*For correspondence: madan@ncbs.res.in (MR)

5 Alkesh Yadav ${ }^{1}$, Quentin Vagne ${ }^{2}$, Pierre Sens $^{2}$, Garud lyengar ${ }^{3}$, Madan Rao $^{4}$

6 ${ }^{1}$ Raman Research Institute, Bangalore 560080, India; ${ }^{2}$ Laboratoire Physico Chimie Curie, 7 Institut Curie, CNRS UMR168, 75005 Paris, France; ${ }^{3}$ Industrial Engineering and

\& Operations Research, Columbia University, New York 10027, USA; ${ }^{4}$ Simons Centre for

- the Study of Living Machines, National Centre for Biological Sciences (TIFR), Bangalore

10560065 , India

12

13

14

15

16

17

18

19

20

21

\begin{abstract}
Many proteins that undergo sequential enzymatic modification in the Golgi cisternae are displayed at the plasma membrane as cell identity markers. The modified proteins, called glycans, represent a molecular code. The fidelity of this glycan code is measured by how accurately the glycan synthesis machinery realises the desired target glycan distribution for a particular cell type and niche. In this paper, we construct a simplified chemical synthesis model to quantitatively analyse the tradeoffs between the number of cisternae, and the number and specificity of enzymes, required to synthesize a prescribed target glycan distribution of a certain complexity to within a given fidelity. We find that to synthesize complex distributions, such as those observed in real cells, one needs to have multiple cisternae and precise enzyme partitioning in the Golgi. Additionally, for fixed number of enzymes and cisternae, there is an optimal level of specificity (promiscuity) of enzymes that achieves the target distribution with high fidelity. The geometry of the fidelity landscape in the multidimensional space of the number and specificity of enzymes, inter-cisternal transfer rates, and number of cisternae, provides a measure for robustness and identifies stiff and sloppy directions. Our results show how the complexity of the target glycan distribution and number of glycosylation enzymes places functional constraints on the Golgi cisternal number and enzyme specificity.
\end{abstract}

\section{Introduction}

A majority of the proteins synthesized in the endoplasmic reticulum (ER) are transferred to the Golgi cisternae for further chemical modification by glycosylation (Alberts et al., 2002), a process that sequentially and covalently attaches sugar moieties to proteins, catalyzed by a set of enzymatic reactions within the ER and the Golgi cisternae. These enzymes, called glycosyltransferases, are localized in the ER and cis-medial and trans Golgi cisternae in a specific manner (Varki et al., 
2009; Cummings and Pierce, 2014). Glycans, the final products of this glycosylation assembly line are delivered to the plasma membrane (PM) conjugated with proteins, whereupon they engage in multiple cellular functions, including immune recognition, cell identity markers, cell-cell adhesion and cell signalling (Varki et al., 2009; Cummings and Pierce, 2014; Varki, 2017; Drickamer and Taylor, 1998; Gagneux and Varki, 1999). This glycan code (Gabius, 2018; Dwek, 1996), representing information (Winterburn and Phelps, 1972) about the cell, is generated dynamically, following the biochemistry of sequential enzymatic reactions and the biophysics of secretory transport (Varki, 2017, 1998; Pothukuchi et al., 2019).

In this paper, we will focus on the role of glycans as markers of cell identity. For the glycans to play this role, they must inevitably represent a molecular code (Gabius, 2018; Varki, 2017; Pothukuchi et al., 2019). While the functional consequences of glycan alterations have been well studied, the glycan code has remained an enigma (Gabius, 2018; Pothukuchi et al., 2019; Bard and Chia, 2016; D'Angelo et al., 2013). We study the fidelity of molecular code generation, i.e. the precision and reliability with which the glycan distribution is created. While it has been recognised that fidelity of the glycan code is necessary for reliable cellular recognition (Demetriou et al., 2001), a quantitative measure of fidelity of the mechanism, and the constraints that fidelity requirements put on cellular structure and organization are lacking.

There are two aspects of the cell-type specific glycan code and the code generation mechanism that have an important bearing on quantifying fidelity. The first is that extant glycan distributions have high complexity (Sect. 2), owing to evolutionary pressures arising from (a) reliable cell type identification amongst a large set of different cell types in a complex organism, the preservation and diversification of "self-recognition" (Drickamer and Taylor, 1998), (b) pathogen-mediated selection pressures (Varki et al., 2009; Varki, 2017; Gagneux and Varki, 1999), and (c) herd immunity within a heterogenous population of cells of a community (WILLS and GREEN, 1995) or within a single organism (Drickamer and Taylor, 1998). We interpret this to mean that the target distribution of glycans for a given cell type is complex; in Sect. 2 we define a quantitative measure for complexity and demonstrate its implications. The second is that the cellular machinery for the synthesis of glycans, which involves sequential chemical processing via cisternal resident enzymes and cisternal transport, is subject to variation and noise (Varki, 2017, 1998; Pothukuchi et al., 2019); the synthesized glycan distribution is, therefore, a function of cellular parameters such as the number and specificity of enzymes, inter-cisternal transfer rates, and number of cisternae. We will discuss an explicit model of the cellular synthesis machinery in Sect. 3.

Here we define fidelity as the minimum achievable Kullback-Leibler $(\mathrm{KL})$ divergence (Cover and Thomas, 2012; MacKay, 2003) between the synthesized distribution of glycans and the target glycan distribution as function of given cellular parameters, such as the number and specificity of enzymes, inter-cisternal transfer rates, and number of cisternae (Sect. 5). Using a simplified chemical synthesis model, we analyze the tradeoffs between the number of cisternae and the number and specificity of enzymes, in order to achieve a prescribed target glycan distribution with high fidelity (Sect. 6). Our analysis leads to a number of interesting results, a few of which we list here:

(i) First, since an important function of the glycan spectrum is cell type/niche identification, it seems natural to relate glycan complexity to organismal complexity taken to be associated with the number of cell types in the organism (Carroll, 2001; Bonner, 1998). Here, we provide a measure of the complexity of the glycan distribution of a given cell type using MSMS data. Using this we have analysed the MSMS data from Hydra, Planaria, Mammalian cells. We find that the complexity of the glycan distribution indeed correlates with the organism complexity.

(ii) Constructing a high fidelity representation of a complex target distribution, such as those observed in real cells, requires a complex golgi machinery with multiple cisternae, precise enzyme 
partitioning and control on enzyme specificity. This definition of fidelity of the glycan code, allows us to provide a quantitative argument for the evolutionary requirement of multiplecompartments. While it is possible to produce complex glycan distributions in one compartment using a large number of enzymes, such a design would inevitably require a more elaborate genetic cost.

(iii) Within our synthesis model, an increase in the number of Golgi cisternae drives an increase in the glycan complexity, keeping everything else fixed.

(iv) We explore the geometry of the fidelity landscape in the multidimensional space of the number and specificity of enzymes, inter-cisternal transfer rates, and number of cisternae. This allows us to to discuss issues such as robustness to noise, and stiff and sloppy directions in this multidimensional space.

(v) For fixed number of enzymes and cisternae, there is an optimal level of specificity of enzymes that achieves the complex target distribution with high fidelity. Keeping the number of enzymes fixed, having low specificity or sloppy enzymes and larger cisternal number could give rise to a diverse repertoire of functional glycans, a strategy used in organisms such as plants and algae. Promiscuous enzymes bring in the potential for evolvability (Kirschner and Gerhart, 2008), promiscuity allows the system to be stable to random mutations in proteins or variations in the target distribution.

Thus, our results imply that the pressure to produce the target glycan code for a given cell type with high fidelity, places strong constraints on the cisternal number and enzyme specificity (Sengupta and Linstedt, 2011). Taken together, our quantitative analysis of the tradeoffs has deep implications for the nonequilibrium self assembly of Golgi cisternae, and suggests that the control of cisternal number must involve a coupling of nonequilibrium self assembly of cisternae with enzymatic chemical reaction kinetics (Glick and Malhotra, 1998). This combined dynamics of chemical processing with nonequilibrium membrane dynamics involving fission, fusion and transport (Sachdeva et al., 2016; Sens and Rao, 2013), opens up a new direction for future research.

\section{Complexity of glycan code}

Since each cell type (in a niche) is identified with a distinct glycan profile (Gabius, 2018; Varki, 2017; Pothukuchi et al., 2019), and this glycan profile is noisy because of the stochastic noise associated with the synthesis and transport (Pothukuchi et al., 2019; Bard and Chia, 2016; D'Angelo et al., 2013), a large number of different cell types can be differentiated only if the cells are able to produce a large set of glycan profiles that are distinguishable in the presence of this noise. Our task is to identify a quantitative measure for the complexity of a glycan profile such that a set of more complex glycan profiles is able to support a larger number of well separated profiles, and therefore, a larger number cell types, or equivalently, a more complex organism ${ }^{1}$. Furthermore, we would like to be able to estimate the complexity of a glycan profile from molecular structure (MSMS) measurements (Cummings and Crocker, 2020; Subramanian et al., 2018; Sahadevan et al., 2014).

In order to identify such a quantitative measure of complexity, we first need a consistent way of smoothening or coarse-graining the the raw glycan profiles obtained from MSMS measurements to remove measurement and synthesis noise. Here, we denoise the glycan profile by approximating it by a Gaussian mixture model (GMM) with specified number of components that are supported on a finite set of indices (Bacharoglou, 2010). Since the size of the set of all possible $m$-component Gaus-

\footnotetext{
${ }^{1}$ A rigorous definition of complexity can be given in terms of the Kullback-Leibler metric Cover and Thomas (2012); MacKay (2003) between two glycan profiles. We declare that two profiles are distinguishable only if the Kullback-Leibler distance between the profiles is more than a given tolerance. This tolerance is an increasing function of the noise. We define the complexity of a set of possible glycan profiles as the size of the largest subset such that the Kullback-Leibler distance of any pair of profiles is larger than the tolerance.
} 
(a)

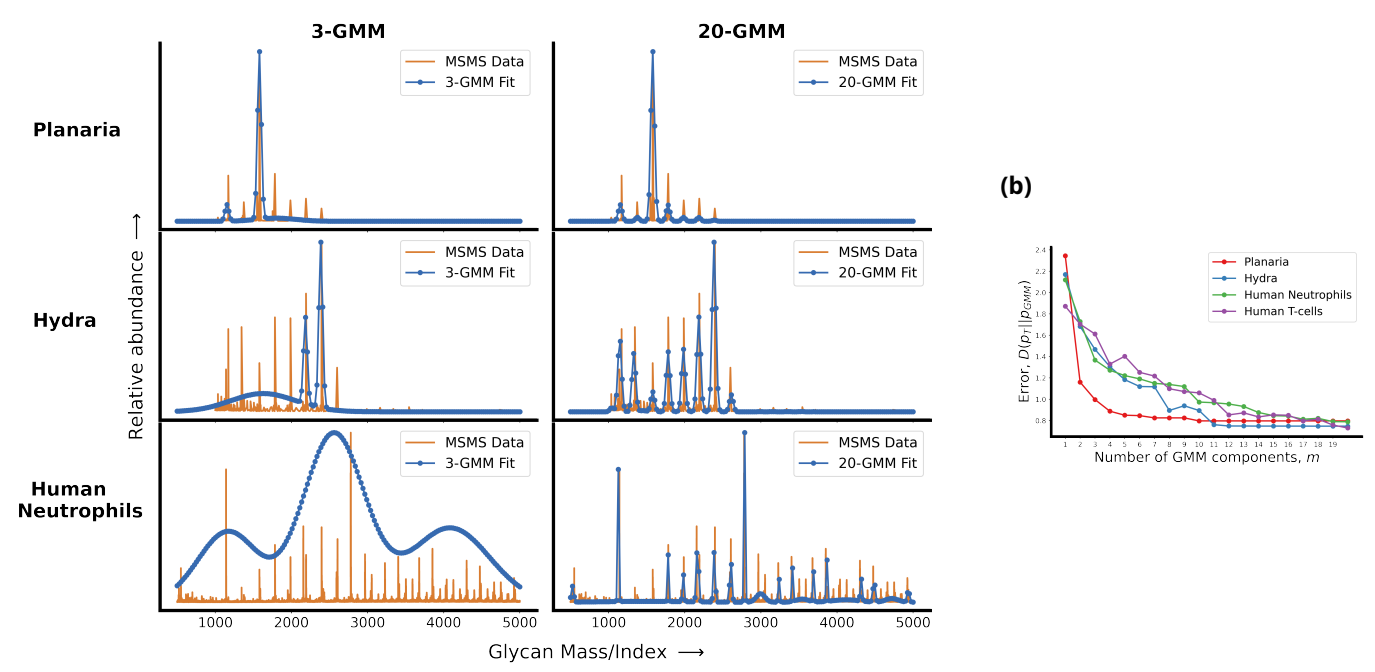

Figure 1. Living cells display a complex glycan distribution. (a) 3-GMM and 20-GMM approximation for the relative abundance of glycans taken from MSMS data of planaria S.mediterranea, hydra magnipapillata and human Neutrophils. (b) The change in the KL-divergence $D\left(p_{T} \| p_{G M M}^{(m)}\right)$ as a function of the number of GMM components $m$. The KL-divergence for planaria saturates at $m=5$, for hydra at $m=11$, and for human cells at $m=20$. Thus, the number of components required to approximate the glycan profile correlates well with the complexity of the organism. Details appear in Appendix 1

sian densities is an increasing function of $m$, we define the complexity of a mixture of Gaussians as the number of components $m$. Figure 1 demonstrates that the value of $m$ at which the $m$-component GMM approximation of the target profile saturates, is a good measure of complexity. Using this definition we see that the complexity of the glycan profiles of various organisms correlates well with the number of cell types in an organism (details of the procedure are in Appendix 1). We will now describe a general model of the cellular machinery that is capable of synthesizing glycans of any complexity. We expect that cells need a more elaborate mechanism to produce profiles from a more complex set.

\section{Synthesis of glycans in the Golgi cisternae}

The glycan display at the cell surface is a result of proteins that flux through and undergo sequential chemical modification in the secretory pathway, comprising an array of Golgi cisternae situated between the ER and the PM, as depicted in Fig. 2. Glycan-binding proteins (GBPs) are delivered from the ER to the first cisterna, whereupon they are processed by the resident enzymes in a sequence of steps that constitute the $\mathrm{N}$-glycosylation process (Varki et al., 2009). A generic enzymatic reaction in the cisterna involves the catalysis of a group transfer reaction in which the monosaccharide moiety of a simple sugar donor substrate, e.g. UDP-Gal, is transferred to the acceptor substrate, by a Michaelis-Menten (MM) type reaction (Varki et al., 2009)

$$
\begin{aligned}
& \text { Acceptor + glycosyl donor + Enzyme } \\
& \qquad \underset{\omega_{b}}{\stackrel{\omega_{f}}{\rightleftharpoons}}[\text { Acceptor } \cdot \text { glycosyl donor } \cdot \text { Enzyme }] \stackrel{\omega_{c}}{\longrightarrow} \text { glycosylated acceptor + nucleotide + Enzyme }
\end{aligned}
$$

From the first cisterna, the proteins with attached sugars are delivered to the second cisterna at a given inter-cisternal transfer rate, where further chemical processing catalysed by the enzymes resident in the second cisterna occurs. This chemical processing and inter-cisternal transfer continues until the last cisterna, thereupon the fully processed glycans are displayed at the PM (Varki 


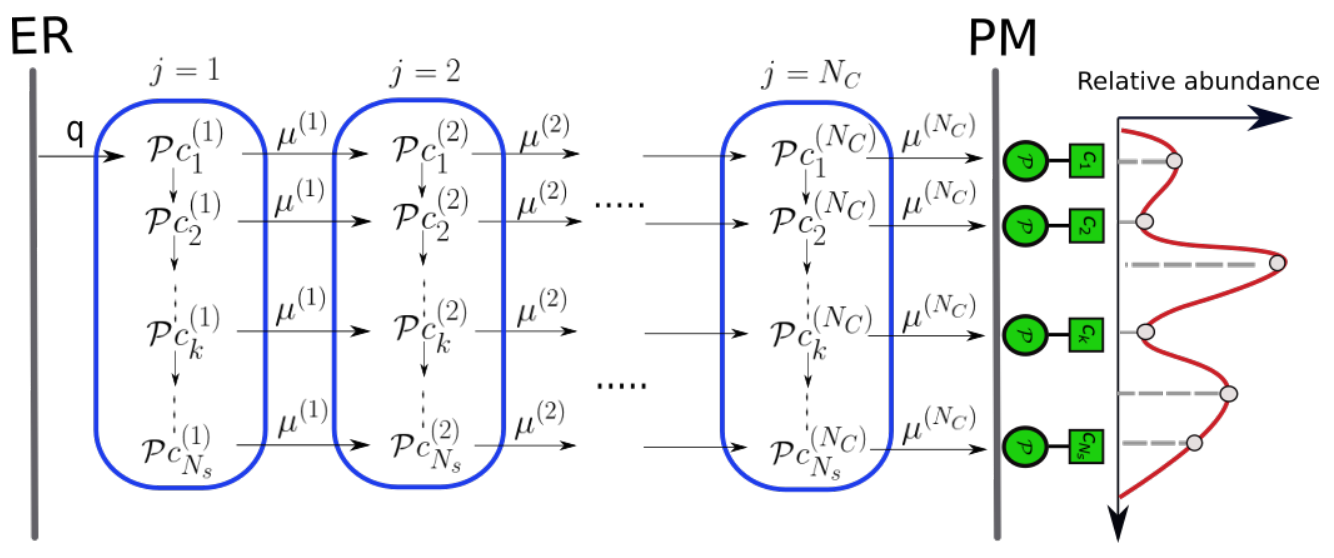

Figure 2. Enzymatic reaction and transport network in the secretory pathway. Represented here is the array of Golgi cisternae (blue) indexed by $j=1, \ldots, N_{C}$ situated between the ER and PM. Glycan-binding proteins $\mathcal{P} c_{1}^{(1)}$ are injected from the ER to cisterna-1 at rate $q$. Superimposed on the Golgi cisternae is transition network of chemical reactions (column) - intercisternal transfer (rows), the latter with rates $\mu^{(j)}$. $\mathcal{P} c_{k}^{(j)}$ denotes the acceptor substrate in compartment $j$ and the glycosyl donor $c_{0}$ is chemostated in each cisterna. This results in a distribution (relative abundance) of glycans displayed at the PM (red curve), that is representative of the cell type.

et al., 2009). The network of chemical processing and inter-cisternal transfer forms the basis of the physical model that we will describe next.

Any physical model of such a network of enzymatic reactions and cisternal transfer needs to be augmented by reaction and transfer rates and chemical abundances. To obtain the range of allowed values for the reaction rates and chemical abundances, we use the elaborate enzymatic reaction models, such as the KB2005 model (Umaña and Bailey, 1997; Krambeck et al., 2009; Krambeck and Betenbaugh, 2005) (with a network of 22, 871 chemical reactions and 7,565 oligosaccharide structures) that predict the $\mathrm{N}$-glycan distribution based on the activities and levels of processing enzymes distributed in the Golgi-cisternae of mammalian cells. For the allowed rates of cisternal transfer, we rely on the recent study by Ungar and coworkers (Fisher et al., 2019; Fisher and Ungar, 2016), whose study shows how the overall Golgi transit time and cisternal number, can be tuned to engineer a homogeneous glycan distribution.

\section{Model for glycan synthesis}

\subsection{Chemical reaction and transport network in cisternae}

We consider an array of $N_{C}$ Golgi cisternae, labelled by $j=1, \ldots, N_{C}$, between the ER and the PM (Fig. 2). Glycan-binding proteins (GBPs), denoted as $\mathcal{P} c_{1}^{(1)}$, are delivered from the ER to cisterna1 at an injection rate $q$. It is well established that the concentration of the glycosyl donor in the $j$-th cisterna is chemostated (Varki et al., 2009; Hirschberg et al., 1998; Caffaro and Hirschberg, 2006; Berninsone and Hirschberg, 2000), thus in our model we hold its concentration $c_{0}^{(j)}$ constant in time for the $j$-th cisterna. The acceptor $\mathcal{P} c_{1}^{(1)}$ reacts with $c_{0}^{(1)}$ to form the glycosylated acceptor $\mathcal{P} c_{2}^{(1)}$, following an MM-reaction (1) catalysed by the appropriate enzyme. The acceptor $\mathcal{P} c_{2}^{(1)}$ has the potential of being transformed into $\mathcal{P}_{3}^{(1)}$, and so on, provided the requisite enzymes are present in that cisterna. This leads to the sequence of enzymatic reactions $\mathcal{P} c_{1}^{(1)} \rightarrow \mathcal{P} c_{2}^{(1)} \rightarrow \ldots \mathcal{P} c_{k}^{(1)} \rightarrow \ldots$, where $k$ enumerates the sequence of glycosylated acceptors, using a consistent scheme (such as in Umaña and Bailey (1997)). The glycosylated GBPs are transported from cisterna-1 to cisterna-2 at an inter-cisternal transfer rate $\mu^{(1)}$, whereupon similar enzymatic reactions proceed. The processes 
of intra-cisternal chemical reactions and inter-cisternal transfer continue to the other cisternae and form a network as depicted in Fig. 2. Although, in this paper, we focus on a sequence of reactions that form a line-graph, the methodology we propose extends to tree-like reaction sequences, and more generally to reaction sequences that form a directed acyclic graphs (Trinajstic, 2018).

Let $N_{s}-1$ denote the maximum number of possible glycosylation reactions in each cisterna $j$, catalysed by enzymes labelled as $E_{\alpha}^{(j)}$, with $\alpha=1, \ldots, N_{E^{\prime}}$, where $N_{E}$ is the total number of enzyme species in each cisterna. Since many substrates can compete for the substrate binding site on each enzyme, one expects in general that $N_{s} \gg N_{E}$. The configuration space of the network in Fig. 2 is $N_{s} \times N_{C}$. For the N-glycosylation pathway in a typical mammalian cell, $N_{s}=2 \times 10^{4}, N_{E}=10-20$ and $N_{C}=4-8$ (Umaña and Bailey, 1997; Krambeck and Betenbaugh, 2005; Krambeck et al., 2009; Fisher and Ungar, 2016). We account for the fact that the enzymes have specific cisternal localisation, by setting their concentrations to zero in those cisternae where they are not present.

The action of enzyme $E_{\alpha}^{(j)}$ on the substrate $\mathcal{P} c_{k}^{(j)}$ in cisterna $j$ is given by

$$
\mathcal{P} c_{k}^{(j)}+E_{\alpha}^{(j)} \underset{\omega_{b}(j, k, \alpha)}{\stackrel{\omega_{f}(j, k, \alpha) c_{0}^{(j)}}{\rightleftharpoons}}\left[E_{\alpha}^{(j)}-\mathcal{P} c_{k}^{(j)}-c_{0}^{(j)}\right] \stackrel{\omega_{c}(j, k, \alpha)}{\longrightarrow} \mathcal{P} c_{k+1}^{(j)}+E_{\alpha}^{(j)}
$$

where $k=1, \ldots N_{s}-1$. In general, the forward, backward and catalytic rates $\omega_{f}, \omega_{b}$ and $\omega_{c}$, respectively, depend on the cisternal label $j$, the reaction label $k$, and the enzyme label $\alpha$, that parametrise the MM-reactions (Price and Stevens, 1999). For instance, structural studies on glycosyltransferasemediated synthesis of glycans (Moremen and Haltiwanger, 2019), would suggest that the forward rate $\omega_{f}$ to depend on the binding energy of the enzyme $E_{\alpha}^{(j)}$ to acceptor substrate $\mathcal{P} c_{k}^{(j)}$ and a physical variable that characterises the cisternae.

A potential candidate for such a cisternal variable is $\mathrm{pH}$ (Kellokumpu, 2019), whose value is maintained homeostatically in each cisterna (Casey et alo, 2010); changes in pH can affect the shape of an enzyme (substrate) or their charge properties, and in general the reaction efficiency of an enzyme has a pH optimum (Price and Stevens, 1999). Another possible candidate for a cisternal variable is membrane bilayer thickness (Dmitrieff et al., 2013) - indeed both pH (Llopis et al., 1998) and membrane thickness are known to have a gradient across the Golgi cisternae. We take $\omega_{f}(j, k, \alpha) \propto P^{(j)}(k, \alpha)$, where $P^{(j)}(k, \alpha) \in(0,1)$, is the binding probability of enzyme $E_{\alpha}^{(j)}$ with substrate $\mathcal{P} c_{k}^{(j)}$, and define the binding probability $P^{(j)}(k, \alpha)$ using a biophysical model, similar in spirit to the Monod-Wyman-Changeux model of enzyme kinetics (Monod et al., 1965; Changeux and Edelstein, 2005) that depends on enzyme-substrate induced fit.

Let $\boldsymbol{\ell}_{\alpha}^{(j)}$ and $\boldsymbol{\ell}_{k}$ denote, respectively, the optimal "shape" for enzyme $E_{\alpha}^{(j)}$ and the substrate $\mathcal{P} c_{k}^{(j)}$. We assume that the mismatch (or distortion) energy between the substrate $k$ and enzyme $E_{\alpha}^{(j)}$ is $\left\|\boldsymbol{\ell}_{k}-\boldsymbol{\ell}_{\alpha}^{(j)}\right\|$, with a binding probability given by,

$$
P^{(j)}(k, \alpha)=\exp \left(-\sigma_{\alpha}^{(j)}\left\|\boldsymbol{\ell}_{k}-\boldsymbol{\ell}_{\alpha}^{(j)}\right\|\right)
$$

where $\|\cdot\|$ is a distance metric defined on the space of $\ell_{\alpha}^{(j)}$ (e.g., the square of the $\ell_{2}$-norm would be related to an elastic distortion model (Savir and Tlusty, 2007)) and the vector $\sigma \equiv\left[\sigma_{\alpha}^{(j)}\right]$ parametrises enzyme specificity. This distortion model captures the above idea that the reaction between the flexible enzyme and fixed substrate is facilitated by an induced fit. A large value of $\sigma_{\alpha}^{(j)}$ indicates a highly specific enzyme, a small value of $\sigma_{\alpha}^{(j)}$ indicates a promiscuous enzyme. It is recognised that the degree of enzyme specificity or sloppiness is an important determinant of glycan distribution (Varki et al., 2009; Roseman, 2001; Hossler et al., 2007; Yang et al., 2018).

Our synthesis model is mean-field, in that we ignore stochasticity in glycan synthesis that may arise from low copy numbers of substrates and enzymes, multiple substrates competing for the same enzymes, and kinetics of inter-cisternal transfer (Umaña and Bailey, 1997; Krambeck et al., 
2009; Krambeck and Betenbaugh, 2005). Then the usual MM-steady state conditions for (2), which assumes that the concentration of the intermediate enzyme-substrate complex does not change with time, imply that

$$
\left[E_{\alpha}^{(j)}-\mathcal{P} c_{k}^{(j)}-c_{0}^{(j)}\right]=\frac{\omega_{f}(j, k, \alpha) c_{0}^{(j)}}{\omega_{b}(j, k, \alpha)+\omega_{c}(j, k, \alpha)} E_{\alpha}^{(j)} c_{k}^{(j)} .
$$

where $c_{k}^{(j)}$ is the concentration of the acceptor substrate $\mathcal{P} c_{k}^{(j)}$ in compartment $j$.

Together with the constancy of the total enzyme concentration, $\left[E_{\alpha}^{(j)}\right]_{t o t}=E_{\alpha}^{(j)}+\sum_{k=1}^{N_{s}}\left[E_{\alpha}^{(j)}-\mathcal{P} c_{k}^{(j)}-c_{0}^{(j)}\right]$, this immediately fixes the kinetics of product formation (not including inter-cisternal transport),

$$
\frac{d c_{k+1}^{(j)}}{d t}=\sum_{\alpha=1}^{N_{E}} \frac{V(j, k, \alpha) P^{(j)}(k, \alpha) c_{k}^{(j)}}{M(j, k, \alpha)\left(1+\sum_{k^{\prime}=1}^{N_{s}} \frac{P^{(j)}\left(k^{\prime}, \alpha\right) c_{k^{\prime}}^{(j)}}{M\left(j, k^{\prime}, \alpha\right)}\right)} \quad k=1, \ldots, N_{S} ; j=1, \ldots, N_{C}
$$

where

$$
M(j, k, \alpha)=\frac{\omega_{b}(j, k, \alpha)+\omega_{c}(j, k, \alpha)}{\omega_{f}(j, k, \alpha) c_{0}^{(j)}} P^{(j)}(k, \alpha)
$$

and

$$
V(j, k, \alpha)=\omega_{c}(j, k, \alpha)\left[E_{\alpha}^{(j)}\right]_{t o t} .
$$

This reparametrization of the reaction rates $\omega_{f}, \omega_{b}, \omega_{c}$ in terms of $\mathbf{M}, \mathbf{V}$ is convenient, since it relates to experimentally measurable parameters $V_{\max }$ and MM-constant $K_{M}$, for each $(j, k, \alpha)$ which can be easily read out (see Appendix 2). As is the usual case, the maximum velocity $V_{\max }$ is not an intrinsic property of the enzyme, because it is dependent on the enzyme concentration $\left[E_{\alpha}^{(j)}\right]_{t o t}$; while $K_{M}(j, k, \alpha)=M(j, k, \alpha) c_{0}^{(j)} / P^{(j)}(k, \alpha)$ is an intrinsic parameter of the enzyme and the enzymesubstrate interaction. The enzyme catalytic efficiency, the so-called " $k_{c a t} / K_{M}$ " $\propto P^{(j)}(k, \alpha)$ and is high for perfect enzymes (Bar-Even et al., 2015) with minimum mismatch.

We now add to this chemical reaction kinetics, the rates of injection $(q)$ and inter-cisternal transport $\mu^{(j)}$ from the cisterna $j$ to $j+1$; in Appendix 3 we display the complete set of equations that describe the changes in the substrate concentrations $c_{k}^{(j)}$ with time. These kinetic equations automatically obey the conservation law for the protein concentration ( $p$ ). At steady state these kinetic equation lead to a set of nonlinear recursion equations (15)-(16) that are displayed in Appendix 3, which can be solved numerically to obtain the steady state glycan concentrations, $\mathbf{c} \equiv c_{k}^{(j)}$, as a function of the independent vectors $\mathbf{M} \equiv[M(j, k, \alpha)], \mathbf{V} \equiv[V(j, k, \alpha)]$, and $\mathbf{L} \equiv\left[P^{(j)}(k, \alpha)\right]$, the transport rates $\boldsymbol{\mu} \equiv\left[\mu^{(j)}\right]$ and specificity, $\boldsymbol{\sigma} \equiv\left[\sigma_{\alpha}^{(j)}\right]$.

\section{Optimization Problem}

Let $c^{*}$ denote the "target" concentration distribution, normalized the distribution so that $\sum_{k=1}^{N_{s}} c_{k}^{*}=1$, for a particular cell type, i.e. the goal of the sequential synthesis mechanism described in Sect. 4.1 is to approximate $c^{*}$. Let $\bar{c}$ denote the normalized steady state glycan concentration distribution displayed on the PM. Then (16) implies that $\bar{c}_{k}=\mu^{\left(N_{C}\right)} c_{k}^{\left(N_{C}\right)}, k=1, \ldots, N_{s}$. We measure the fidelity $F\left(c^{*} \| \overline{\boldsymbol{c}}\right)$ between the $\mathbf{c}^{*}$ and $\overline{\mathbf{c}}$ by the ratio of the Kullback-Leibler divergence $D\left(\boldsymbol{c}^{*} \| \boldsymbol{c}\right)$ (Cover and Thomas, 2012; MacKay, 2003) to the entropy $H\left(c^{*}\right)$

$$
F\left(\boldsymbol{c}^{*} \| \overline{\boldsymbol{c}}\right):=\frac{\boldsymbol{D}\left(\boldsymbol{c}^{*} \| \overline{\mathbf{c}}\right)}{\boldsymbol{H}\left(\boldsymbol{c}^{*}\right)}=\frac{\sum_{k=1}^{N_{s}} c_{k}^{*} \ln \left(\frac{c_{k}^{*}}{\bar{c}_{k}}\right)=\sum_{k=1}^{N_{s}} c_{k}^{*} \ln \left(\frac{c_{k}^{*}}{c_{k}^{\left(N_{C}\right)} \mu^{\left(N_{C}\right)}}\right)}{\sum_{k=1}^{N_{s}} c_{k}^{*} \ln \left(1 / c_{k}^{*}\right)}
$$

The reason why we divide the KL-divergence by the entropy of the target distribution is to enable comparison of the fidelity of the mechanism across target distributions of different complexity. Note that high fidelity corresponds to low values of $F\left(c^{*} \| \bar{c}\right)$, vice versa. 
Thus, the problem of designing a sequential synthesis mechanism that approximates $\mathbf{c}^{*}$ for a given enzyme specificity $\sigma$, transport rate $\mu$, number of enzymes $N_{E}$, and number of cisternae $N_{C}$ is given by

$$
\text { Optimization A : } \quad \bar{D}\left(\sigma, N_{E}, N_{C}, c^{*}\right):=\min _{\boldsymbol{\mu}, \mathbf{M}, \mathbf{V}, \mathbf{L} \geq \mathbf{0}} F\left(\boldsymbol{c}^{*} \| \overline{\boldsymbol{c}}\right),
$$

where we emphasize that the optimum fidelity $\bar{D}\left(\sigma, N_{E}, N_{C}, c^{*}\right)$ is a function of $\left(\sigma, N_{E}, N_{C}, c^{*}\right)$. Note that there is separation of time scales implicit in Optimization A - the chemical kinetics of the production of glycans and their display on the PM happens over cellular time scales, while the issues of tradeoffs and changes of parameters are related to evolutionary timescales.

Optimization A, though well-defined, is a hard problem, since the steady state concentrations (16) are not explicitly known in terms of the parameters $(\boldsymbol{\mu}, \mathbf{M}, \mathbf{V}, \mathbf{L})$. In Appendix 4, we formulate an alternative problem Optimization $B$ in which the steady state concentrations are defined explicitly in terms of a new parameters $\boldsymbol{\mu}, \mathbf{R}$ and $\mathbf{L}$, and prove that Optimization $A$ and Optimization $B$ are exactly equivalent. This is a crucial insight that allows us to obtain all the results that follow. In Appendix 5, we describe the variant of the Sequential Quadratic Programming (SQP) (Boyd and Vandenberghe, 2004), that we use to numerically solve the optimization problem.

\section{Results of optimization}

The dimension of the optimization search space is extremely large $\approx O\left(N_{s} \times N_{E} \times N_{C}\right)$. To make the optimization search more manageable, we make the following simplifying assumptions:

(i) We ignore the $k$-dependence of the vectors $(\mathbf{M}, \mathbf{V})$, or alternatively of $\mathbf{R}$ - see Appendix 4 for details.

(ii) The enzyme-substrate binding probability $P^{(j)}(k, \alpha)$ is still dependent on the substrate $k$. We assume that shape function is a scalar (a length), i.e. $\boldsymbol{l}_{\alpha}^{(j)}=\ell_{\alpha}^{(j)}$. It further simplifies the algebra to assume that the length of the substrates are integer multiples of a basic unit (which we take to be 1), i.e. $\boldsymbol{\ell}_{k}=k$. The norm that appears in (3) is taken to be the absolute value difference $\left|l_{k}-l_{\alpha}^{(j)}\right|$. Other metrics, such as $\left|l_{k}-l_{\alpha}^{(j)}\right|^{2}$, corresponding to the elastic distortion model (Savir and Tlusty, 2007), do not pose any computational difficulties, and we see that the results of our optimization remain qualitatively unchanged.

(iii) We drop the dependence of the specificity on $\alpha$ and $j$, and take it to be a scalar $\sigma$.

These restrictions significantly reduce the dimension of the optimization search, so much so that in certain limits, we can solve the problem analytically². This helps us obtain some useful heuristics (Appendix 6) on how to tune the parameters, e.g. $N_{E}, N_{C}, \sigma$, and others, in order to generate glycan distributions $\mathbf{c}$ of a given complexity. These heuristics inform our more detailed optimization using "realistic" target distributions.

The calculations in Appendix 6 imply, as one might expect, that the synthesis model needs to be more elaborate, i.e., needs a larger number of cisternae $N_{C}$ or a larger number of enzymes $N_{E}$, in order to produce a more complex glycan distribution. For a real cell type in a niche, the specific elaboration of the synthesis machinery, would depend on a variety of control costs associated with increasing $N_{E}$ and $N_{C}$. While an increase in the number of enzymes would involve genetic

\footnotetext{
${ }^{2}$ In Appendix 6 we show that (21) can be solved analytically in the limit $N_{s} \gg 1$, since the glycan index $k$ can be approximated by a continuous variable, and the recursion relations for the steady state glycan concentrations (15)-(16) can be cast as a matrix differential equation. This allows us to obtain an explicit expression for the steady state concentration in terms of the parameters $(\mathbf{R}, \mathbf{L})$.
} 
and transcriptional costs, the costs involved in increasing the number of cisternae could be rather subtle.

Notwithstanding the relative control costs of increasing $N_{E}$ and $N_{C}$, it is clear from the special case, that increasing the number of cisternae achieves the goal of obtaining an accurate representation of the target distribution. Suppose the target distribution $c_{k}^{*}=\delta(k-M)$ for a fixed $M \gg 1$, i.e. $c_{k}^{*}=1$ when $k=M$, and 0 otherwise, and that the $N_{E}$ enzymes that catalyse the reactions are highly specific. In this limit, Optimization $A$ reduces to a simple enumeration exercise Uaiman and Thattai, 2018): clearly, one needs $N_{E}=M$ enzymes, one for each $k=1, \ldots, M$ reactions, in order to generate $\mathcal{P}_{c_{M}}$. For a single Golgi cisterna with a finite cisternal residence time (finite $\mu$ ), the chemical synthesis network will generate a significant steady state concentration of lower index glycans $\mathcal{P}_{k}$ with $k<M$, contributing to a low fidelity. To obtain high fidelity, one needs multiple Golgi cisternae with a specific enzyme partitioning $\left(E_{1}, E_{2}, \ldots, E_{M}\right)$ with $E_{j}$ enzymes in cisterna $j=1, \ldots, N_{c}$. This argument can be generalized to the case where the target distribution is a finite sum of deltafunctions. The more general case, where the enzymes are allowed to have variable specificity, needs a more detailed study, to which we turn to next.

\subsection{Target distribution from coarse-grained MSMS}

As discussed in Sect. 2, we obtain the target glycan distribution from glycan profiles for real cells using Mass Spectrometry coupled with determination of molecular structure (MSMS) measurements (Cummings and Crocker, 2020). The raw MSMS data, however, is not suitable as a target distribution. This is because it is very noisy, with chemical noise in the sample and Poisson noise associated with detecting discrete events being the most relevant (Du et al., 2008). This means that many of the small peaks in the raw data are not part of the signal, and one has to "smoothen" the distribution to remove the impact of noise.

We use MSMS data from human T-cells (Cummings and Crocker, 2020) for our analysis. As discussed in Sect. 2, the Gaussian mixture models (GMM) are often used to approximate distributions with a mixed number of modes or peaks (MacKay, 2003), or in our setting, a given fixed complexity. Here, we use a variation of the Gaussian mixture models (see Appendix 1 for details) to create a hierarchy of increasingly complex distributions to approximate the MSMS raw data. Thus, the 3-GMM and 20-GMM approximations represent the low and high complexity benchmarks, respectively. In Appendix 1, we show that the likelihood for the glycan distribution of the human T-cell saturates at 20 peaks. Thus, statistically the human T-cell glycan distribution is accurately approximated by 20 peaks.

This hierarchy allows us to study the trade-off between the complexity of the target distribution and the complexity of the synthesis model needed to generate the distribution as follows. Let $\mathbf{T}^{(i)}$ denote the $i$-component GMM approximation for the human T-cell MSMS data. We sample this target distribution at indices $k=1, \ldots, N_{s^{\prime}}$ that represent the glycan indices, and then renormalize to obtain the discrete distribution $\left\{T_{k}^{(i)}, k=1, \ldots, N_{s}\right\}$. To highlight the role of target distribution complexity, we focus on the 3-GMM $\boldsymbol{T}^{(3)}$ (low complexity) and 20-GMM approximation $\boldsymbol{T}^{(20)}$ (high complexity) in the describing our results.

\subsection{Tradeoffs between number of enzymes, number of cisternae and enzyme speci- ficity to achieve given complexity}

We summarize the main results that follow from an optimization of the parameters of the glycan synthesis machinery to a given target distribution in Figs. 3-4. 
(a) Less complex target, 3-GMM approximation

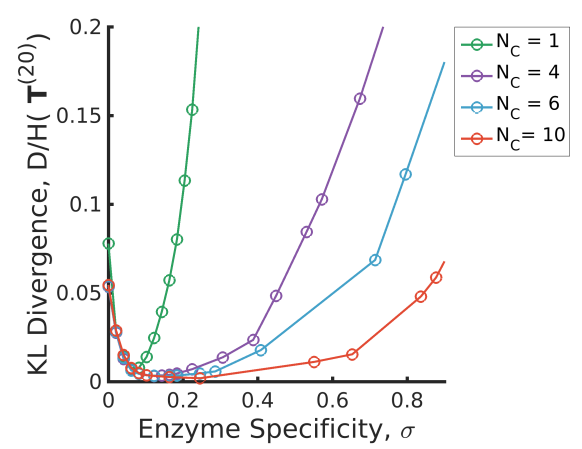

(c) Less complex target, 3-GMM approximation

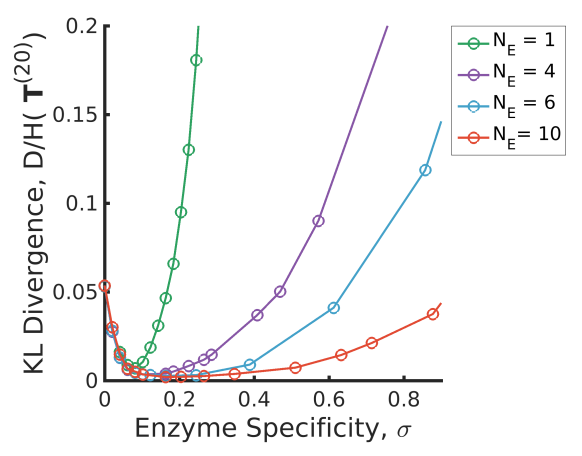

(b) More complex target, 20-GMM approximation

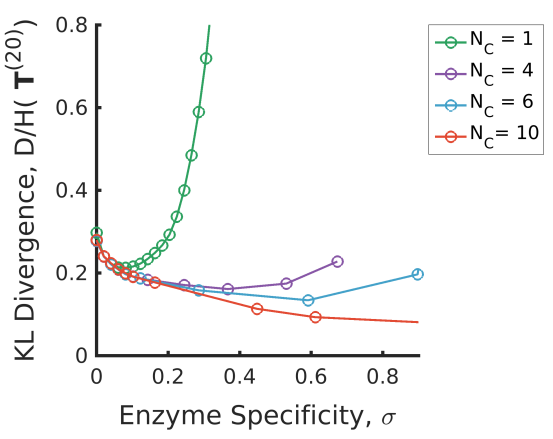

(d) More complex target, 20-GMM approximation

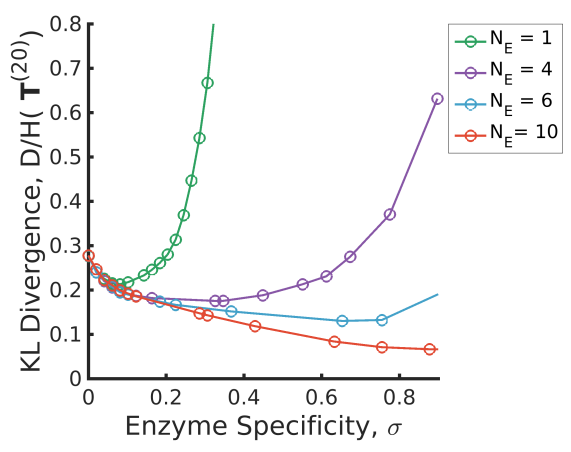

Figure 3. Tradeoffs amongst the glycan synthesis parameters, enzyme specificity $\sigma$, cisternal number $N_{C}$ and enzyme number $N_{E}$, to achieve a complex target distribution $\mathbf{c}^{*}$ ). (a)-(b) Normalised Kullback-Leibler distance $\bar{D}\left(\sigma, N_{E}, N_{C}, \mathbf{c}^{*}\right)$ as function of $\sigma$ and $N_{C}$ (for fixed $N_{E}=3$ ), (c)-(d) $\bar{D}\left(\sigma, N_{E}, N_{C}, \mathbf{c}^{*}\right.$ ) as function of $\sigma$ and $N_{E}$ (for fixed $N_{C}=3$ ), with the target distribution $\mathrm{c}^{*}$ set to the 3-GMM (less complex) and 20-GMM (more complex) approximations for the human T-cell MSMS data. $\bar{D}\left(\sigma, N_{E}, N_{C}, \mathrm{c}^{*}\right)$ is a convex function of $\sigma$ for each $\left(N_{E}, N_{C}, \mathbf{c}^{*}\right)$, decreasing in $N_{C}, N_{E}$ for each $\left(\sigma, \mathbf{c}^{*}\right)$, increasing in the complexity of $\mathbf{c}^{*}$ for fixed $\left(\sigma, N_{E}, N_{C}\right)$. The specificity $\sigma_{\min }\left(\mathbf{c}^{*}, N_{E}, N_{C}\right)=\operatorname{argmin}_{\sigma}\left\{\bar{D}\left(\sigma, N_{E}, N_{C}, \mathbf{c}^{*}\right)\right\}$ that minimises the error for given $\left(N_{E}, N_{C}, \mathbf{c}^{*}\right)$ is an increasing function of $N_{C}, N_{E}$ and the complexity of the target distribution $\mathbf{c}^{*}$. 
(a) The optimal fidelity $\bar{D}\left(\sigma, N_{E}, N_{C}, \mathbf{c}^{*}\right)$ is a convex function of $\sigma$ for fixed values for other parameters (see Fig. 3), i.e. it first decreases with $\sigma$ and then increases beyond a critical value of $\sigma_{\min }$.

The fidelity $\bar{D}\left(\sigma, N_{E}, N_{C}, \mathbf{c}^{*}\right)$ is decreasing in $N_{C}$ and $N_{E}$ for fixed values of the other parameters, and increasing in the complexity of $\mathbf{c}^{*}$ for fixed $\left(\sigma, N_{C}\right)$. The marginal contribution of $N_{C}$ and $N_{E}$ in improving fidelity $\bar{D}$ is approximately equal (see Figs. 4 (a), 4 (b)). We discuss the origin of this symmetry later in this section.

The lower complexity distributions can be synthesized with high fidelity with small $\left(N_{E}, N_{C}\right)$, whereas higher complexity distributions require significantly larger $\left(N_{E}, N_{C}\right)$ (see Figs. 4 (a), 4 (b). For a typical mammalian cell, the number of enzymes in the $\mathrm{N}$-glycosylation pathway are in the range $N_{E}=10-20$ (Umaña and Bailey, 1997; Krambeck and Betenbaugh, 2005; Krambeck et al., 2009; Fisher and Ungar, 2016), Fig. 4 (b) would then suggest that the optimal cisternal number would range from $N_{C}=3-8$ (Sengupta and Linstedt, 2011).

(b) The optimal enzyme specificity $\sigma_{\min }\left(\mathbf{c}^{*}, N_{C}\right)=\operatorname{argmin}_{\sigma}\left\{\bar{D}\left(\sigma, \bar{N}_{E}, N_{C}, \mathbf{c}^{*}\right)\right\}$, that minimises the error as function of $\left(N_{C}, \mathbf{c}^{*}\right)$ with $N_{E}$ fixed at $\bar{N}_{E}$, is an increasing function of $N_{C}$ and the complexity of the target distribution $\mathbf{c}^{*}$ (Figs. 3 (a), 3 (b), 4 (c), 4 (d)). This is consistent with the results in Appendix 6 where we established that the width of the synthesized distribution is inversely dependent on the specificity $\sigma$ : since a GMM approximation with fewer peaks has wider peaks, $\sigma_{\min }$ is low, and vice versa. Similar results hold when $N_{C}$ is fixed at $\bar{N}_{C}$, and $N_{E}$ is varied (see Figs. 3 (c), 3 (d), 4 (c), 4 (d)).

Our results are consistent with those in Fisher et al. (2019). They optimize incoming glycan ratio, transport rate and effective reaction rates in order to synthesize a narrow target distribution centred around a desired glycan. The ability to produce specific glycans without much heterogeneity is an important goal in pharmaceutical industry. They define heterogeneity as the total number of glycans synthesized, and show that increasing the number of compartments $N_{C}$ decreases heterogeneity, and increases the concentration of the specific glycan. They also show that the effect of compartments in reducing heterogeneity cannot be compensated by changing the transport rate. Our results are entirely consistent with theirs - we have shown that $\bar{D}$ decreases as we increase $N_{C}$. Thus, if the target distribution has a single sharp peak, increasing $N_{C}$ will reduce the heterogeneity in the distribution.

We insert an important cautionary note here. It would seem that the results in Fig. 4 imply that there is an approximate $N_{E}-N_{C}$ symmetry in the model, i.e. increasing either $N_{E}$ or $N_{C}$ affects the fidelity, optimal enzyme specificity and the sensitivity in approximately the same way. This would be an erroneous inference, and is a consequence of the distortion model we have used for calculating the binding probabilities of substrates with enzymes. The root cause for this apparent symmetry is that we have allowed for all enzymes to catalyse reactions in all cisternae (albeit with different efficiencies). This symmetry is violated by simply restricting the activity of the enzymes to be dependent on the cisternae. A simple realisation of this in terms of the distortion model is given in Appendix 7.

\subsection{Optimal partitioning of enzymes in cisternae}

Having studied the optimum $N_{E}, N_{C}, \sigma$ to attain a given target distribution with high fidelity, we ask what is the optimal partitioning of the $N_{E}$ enzymes in these $N_{C}$ cisternae? Answering this within the context of our chemical reaction model (Sect. 4.1) requires some care, since it incorporates the following enzymatic features: (a) enzymes with a finite specificity $\sigma$ can catalyse several reactions, although with an efficiency that varies with both the substrate index $k$ and cisternal index $j$, and (b) every enzyme appears in each cisternae; however their reaction efficiencies depend on the 
(a) Fidelity for less complex target, $\mathbf{c}^{*}=3-\mathrm{GMM}$ approximation

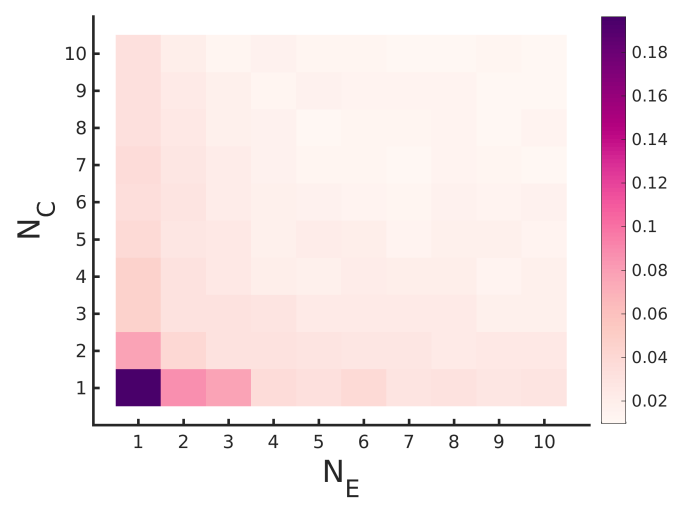

(c) Optimal enzyme specificity for less complex target, $\mathbf{c}^{*}=$ 3-GMM approximation

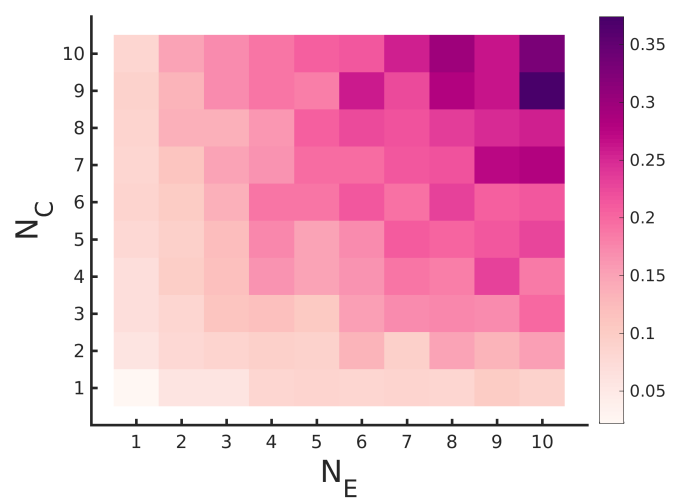

(b) Fidelity for more complex target $\mathbf{c}^{*}=20-\mathrm{GMM}$ approximation

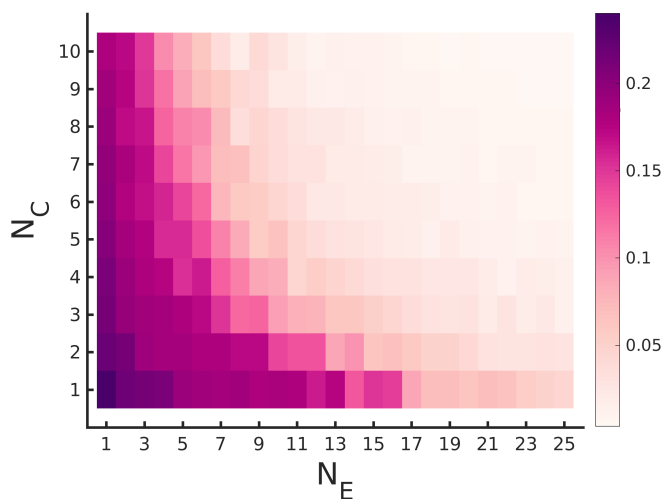

(d) Optimal enzyme specificity for more complex target $\mathbf{c}^{*}=$ 20-GMM approximation

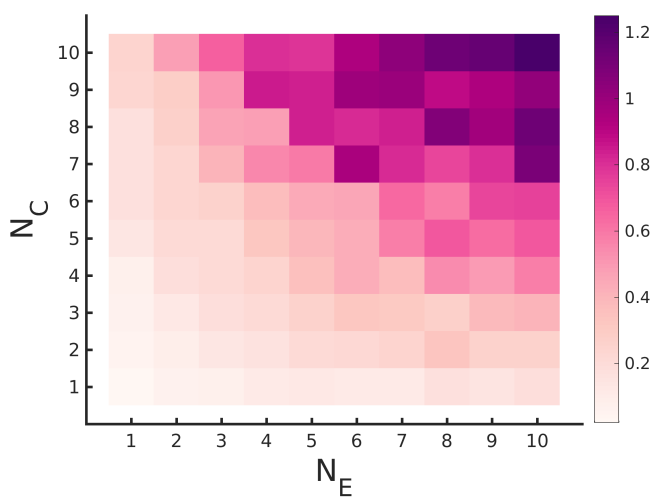

Figure 4. Fidelity of glycan distribution and optimal enzyme properties to achieve a complex target distribution. The target $\mathbf{c}^{*}$ is taken from 3-GMM (less complex) and 20-GMM (more complex) approximations of the human T-cell MSMS data. (a)-(b) Optimum fidelity $\min _{\sigma}\left\{\bar{D}\left(\sigma, N_{C}, N_{E}, \mathbf{c}^{*}\right)\right\}$ as a function of $\left(N_{E}, N_{C}\right)$. More complex distributions require either a larger $N_{E}$ or $N_{C}$. The marginal impact of increasing $N_{E}$ and $N_{C}$ on the fidelity $\bar{D}$ is approximately equal. (c)-(d) Enzyme specificity $\sigma_{\min }$ that achieves $\min _{\sigma}\left\{\bar{D}\left(\sigma, N_{C}, N_{E}, \mathbf{c}^{*}\right)\right\}$ as a function of $\left(N_{E}, N_{C}\right) . \sigma_{\min }$ increases with increasing $N_{E}$ or $N_{C}$. To synthesize the more complex $20 \mathrm{GMM}$ approximation with high fidelity requires enzymes with higher specificity $\sigma_{\min }$ compared to those needed to synthesize the broader, less complex 3-GMM approximation. 
(a)
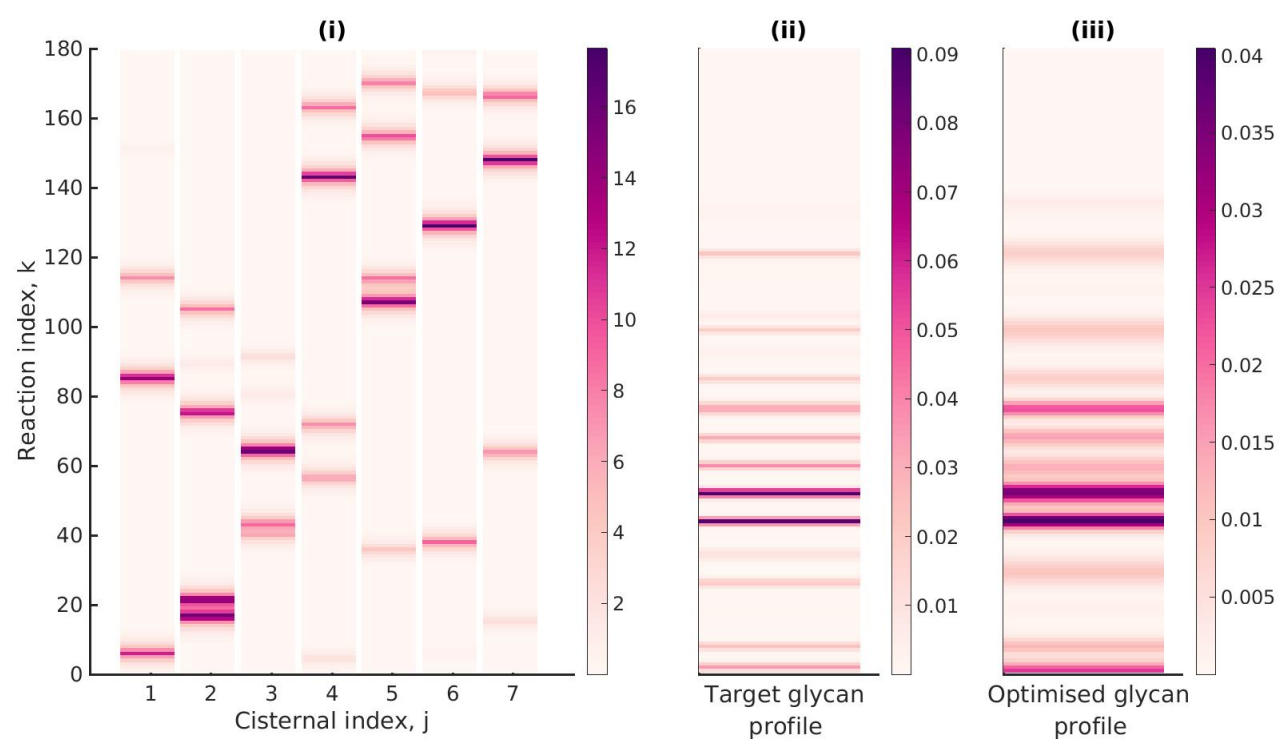

(b)
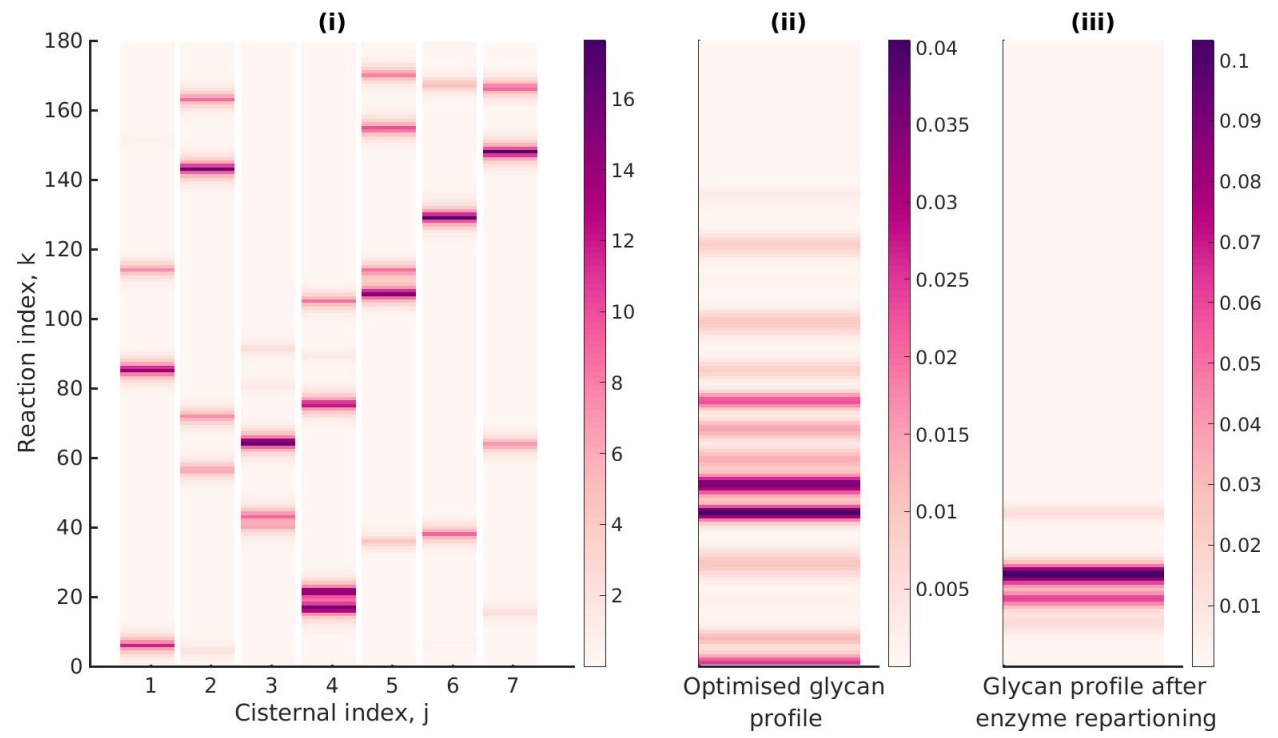

Figure 5. Optimal enzyme partitioning in cisternae. (a) Heat map of the effective reaction rates in each cisterna (representing the optimal enzyme partitioning) and the steady state concentration in the last compartment $\left(\mathbf{c}^{\left(N_{C}\right)}\right)$ for the 20-GMM target distribution. Here $N_{E}=5, N_{C}=7$, normalised $D\left(\mathbf{T}^{(20)} \| \mathbf{c}^{\left(N_{C}\right)}\right) / H\left(\mathbf{T}^{(20)}\right)=0.11$. (b) Effective Reaction rates after swapping the optimal enzymes of the fourth and second cisternae. The displayed glycan profile is considerably altered from the original profile. 
enzyme levels, the enzymatic reaction rates and the enzyme matching function $\mathbf{L}$, all of which depend on the cisternal index $j$.

Therefore, instead of focusing on the cisternal partitioning of enzymes, we identify the chemical reactions that occur with high propensity in each cisternae. For this we define an effective reaction rate $R_{e f f}(j, k)$ for $\mathcal{P}_{k} \rightarrow \mathcal{P} c_{k+1}$ in the $j$-th cisterna as

$$
\boldsymbol{R}_{e f f}(j, k)=\sum_{\alpha=1}^{N_{E}} \boldsymbol{R}_{\alpha}^{(j)} \boldsymbol{P}^{(j)}(k, \alpha) .
$$

According to our model presented in Sect. 4.1, the list of reactions with high effective reaction rates in each cisterna, corresponds to a cisternal partitioning of the perfect enzymes. In a future study, we will consider a Boolean version of a more complex chemical model, to address more clearly, the optimal enzyme partitioning amongst cisternae.

Figure 5 (a) (i) shows the heat map of the effective reaction rates in each cisterna for the optimal $N_{E}, N_{C}, \sigma$ that minimises the normalised KL-distance to the $20 \mathrm{GMM}$ target distribution $\mathbf{T}^{(20)}$ (see Fig. 5 (a) (ii)). The optimized glycan profile displayed in Fig. 5 (a) (iii) is very close to the target. An interesting observation from Fig. 5 (a) (i) is that the same reaction can occur in multiple cisternae.

Keeping everything else fixed at the optimal value, we ask whether simply repartitioning the optimal enzymes amongst the cisternae, alters the displayed glycan distribution. In Fig. 5 (b) (i), we have exchanged the enzymes of the fourth and second cisterna. The glycan profile after enzyme partitioning (see Fig. 5 (b) ((iii)) is now completely altered (compare Fig. 5 (b) (ii) with Fig. 5 (b) (iii)). Thus, one can generate different glycan profiles by repartitioning enzymes amongst the same number of cisternae (Jaiman and Thattai, 2018).

\section{Geometry of the Fidelity landscape}

Here we show that the optimum solution is not unique, rather it is highly degenerate, with several equally good optimum solutions. Thus the multidimensional fidelity landscape in $\mathbf{R}, \boldsymbol{\mu}, \mathbf{L}$ and $\sigma$ is typically rugged. We analyse the geometry of this fitness landscape by doing a local Hessian analysis about the optimal solutions.

\subsection{Degeneracies in the synthesis model}

The synthesis model is highly degenerate, in the sense that many combinations of parameters give rise to the same glycan profile. This makes the optimization non convex as there are many equally good minima. These degeneracies are both discrete and continuous. The continuous degeneracies correspond to regions in reaction rate $(\mathbf{R})$-transport rate $(\boldsymbol{\mu})$ space moving along which does not change the concentration profile. The discrete degeneracies are disconnected regions in the parameter space which correspond to the same glycan profile. The number of discrete degeneracies increases exponentially with increase in $\left(N_{E}, N_{C}\right)$. We also find that the fraction of initial conditions converging to a solution close to the global minima increases on increasing $\left(N_{E}, N_{C}\right)$. Technical details of these issues are discussed in Appendix 8.

\subsection{Stiff and sloppy directions}

We analyze the change in fidelity on small perturbations in $\mathbf{R}, \boldsymbol{\mu}, \mathbf{L}$ and $\sigma$ around the optimal solution. This allows us to determine where the cell needs to develop a tighter control mechanism (stiff directions) and where it has more leeway around the optimal values (sloppy directions). We 
do this by analyzing the eigenvalues and eigenvectors of the Hessian around the optimal point (details in Appendix 9). We find that small perturbations around the optimal values in $\sigma$, change the glycan profile a lot more compared to perturbations in the other parameters and this stiffness in $\sigma$ generally decreases on increasing $N_{E}, N_{C}$ (Fig. 6 (a)-(c)). Small perturbations in $\mu$ and some $\boldsymbol{L}$ directions around the optimum also significantly alter the glycan profile and the stiffness increases on increasing $N_{C}, N_{E}$, eventually becoming comparable to $\sigma$. The glycan profile is robust to perturbations in most $\boldsymbol{R}$ and some $\boldsymbol{L}$ directions (Fig. 6 (b)). The total average stiffness of the optimization parameters, defined by the mean of all eigenvalues of the hessian, decreases on increasing $N_{E}, N_{C}$ (Fig. 6 (c)).

\subsection{Implications for robustness to parametric noise}

Since the synthesized glycan distribution displayed by the cell marks its identity, it must be robust to noise intrinsic to the synthesis machinery. The degeneracy of solutions and sloppy directions in the fidelity landscape makes the glycan distribution robust to intrinsic noise in the synthesis and cell to cell variations in the kinetic parameters. We find that the number of degeneracies increases on increasing $\left(N_{E}, N_{C}\right)$, and the average stiffness of the optimized parameters decreases on increasing $\left(N_{E}, N_{C}\right)$ making the synthesis more robust to parameter fluctuations. Further, while the parameter space is high dimensional, the dimension of controllable parameters (measured by the stiff directions) is low dimensional. We find this dimensional reduction a compelling idea which we will take up later.

\section{Strategies to achieve high glycan diversity}

So far we have studied how the complexity of the target glycan distribution places constraints on the evolution of Golgi cisternal number and enzyme specificity. We now take up another issue, namely, how the physical properties of the Golgi cisternae, namely cisternal number and intercisternal transport rate, may drive diversity of glycans (Varki, 2011; Dennis et al., 2009). There is substantial correlative evidence to support the idea that cell types that carry out extensive glycan processing employ larger numbers of Golgi cisternae. For example, the salivary Brunner's gland cells secrete mucous that contains heavily O-glycosylated mucin as its major component (van Halbeek et al., 1983). The Golgi complex in these specialized cells contain 9 - 11 cisternae per stack. Additionally, several organisms such as plants and algae secrete a rather diverse repertoire of large, complex glycosylated proteins, for a variety of functions (McFarlane et al., 2014; Koch et al., 2015; O'Neill et al., 2004; Hayashi and Kaida, 2011; Kumar et al., 2011; Gow and Hube, 2012; Atmodjo et al., 2013; Free, 2013; Pauly et al., 2013; Burton and Fincher, 2014). These organisms possess enlarged Golgi complexes with multiple cisternae per stack (Becker and Melkonian, 1996; Mironov et al., 2017; Donohoe et al., 2007; Mogelsvang et al., 2003; Ladinsky et al., 2002).

We define diversity as the total number of glycan species produced above a specified threshold abundance $c_{t h}$. This last condition is necessary because very small peaks will not be distinguishable in the presence of noise. In computing the diversity from our chemical synthesis model, we have chosen the threshold to be $c_{t h}=1 / N_{s}$, where $N_{s}$ is the total number of glycan species. We have checked that the qualitative results do not depend on this choice (see Fig. 1 in Appendix 10).

We use the sigmoid function $\left(1+e^{-x / \tau}\right)^{-1}$ as a differentiable approximation to the Heaviside function $\Theta(x)$, define the following optimization to maximize diversity for a given set of parameter values, 
(a)

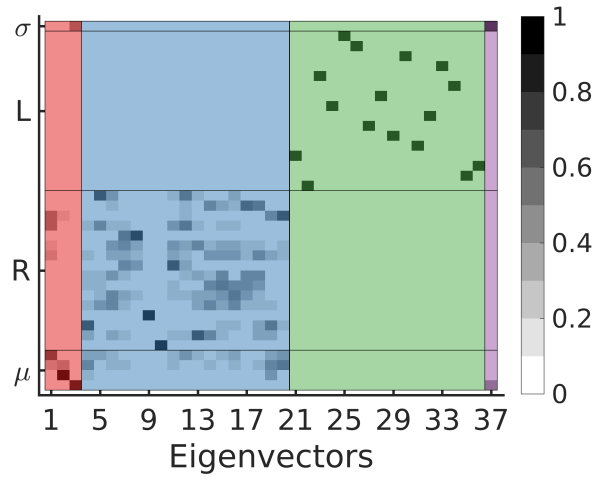

(c)

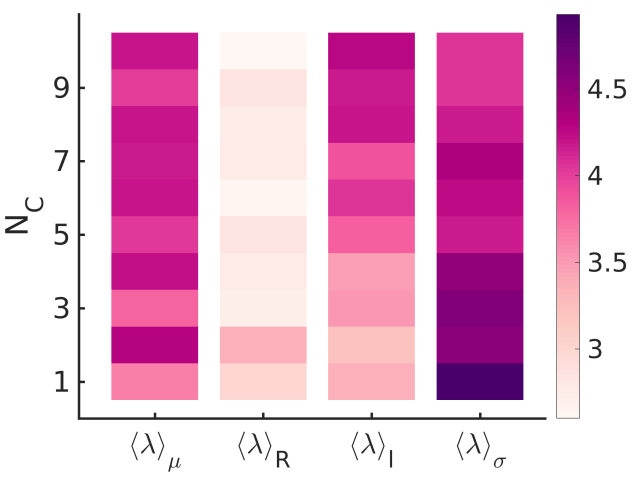

(b)

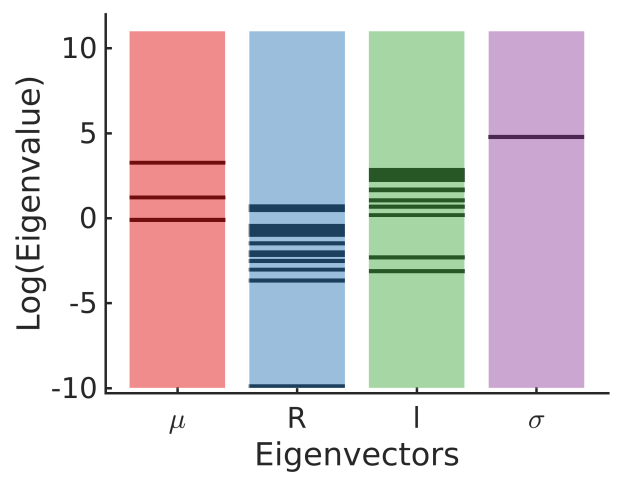

(d)

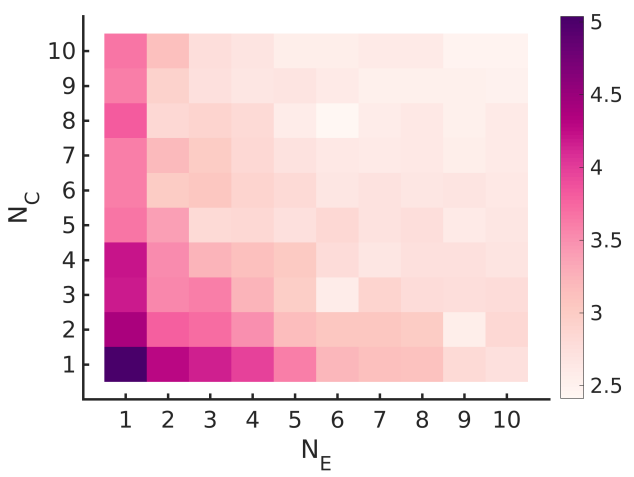

Figure 6. (a) Eigenvectors of the Hessian matrix $\left.\frac{\partial^{2}}{\partial X_{i} \partial X_{j}} F\right|_{X_{\min }}$ for $\left(N_{E}, N_{C}\right)=(4,4)$. The x-axis indexes the $N_{C}+2 N_{E} N_{C}+1=37$ eigenvectors, the y-axis indexes the $N_{C}+2 N_{E} N_{C}+1$ components of the eigenvectors, and the grayscale denotes the absolute value of the component in the range $[0,1]$. The componenets are grouped according to ( $\boldsymbol{\mu}, \boldsymbol{R}, \boldsymbol{L}, \sigma)$ and the eigenvectors are ordered according to the most dominant component in the eigenvector( $\boldsymbol{\mu}$ (orange), $\boldsymbol{R}$ (blue), $\boldsymbol{L}$ (green), $\sigma$ (purple)). There is some mixing of the different components ( $\mathbf{R}$ and $\mu$ or $\sigma$ and $\mu$ ) but this is usually small. (b) The distribution of eigenvalues $\lambda_{i}$ of the Hessian matrix $\left.\frac{\partial^{2}}{\partial X_{i} \partial X_{j}} F\right|_{X_{\min }}$. Each stripe represents an eigenvalue and the location of the stripe on the $\mathrm{x}$-axis represents whether the dominant component of the associated eigenvector belongs to $\mu, \boldsymbol{R}, \boldsymbol{L}$ or $\sigma$ direction. (c) The average stiffness along $\mu, \boldsymbol{R}, \boldsymbol{L}$ or $\sigma$ directions, defined by the log of average of eigenvalues corresponding to the eigenvectors in the respective group, as a function of $N_{C}$ for fixed $N_{E}=4$. (d) Total average stiffness $\langle\lambda\rangle=\log \left(\frac{\sum \lambda_{i}}{N_{C}+2 N_{E} N_{C}+1}\right)$ as a function of $N_{E}, N_{C}$. 
(a)

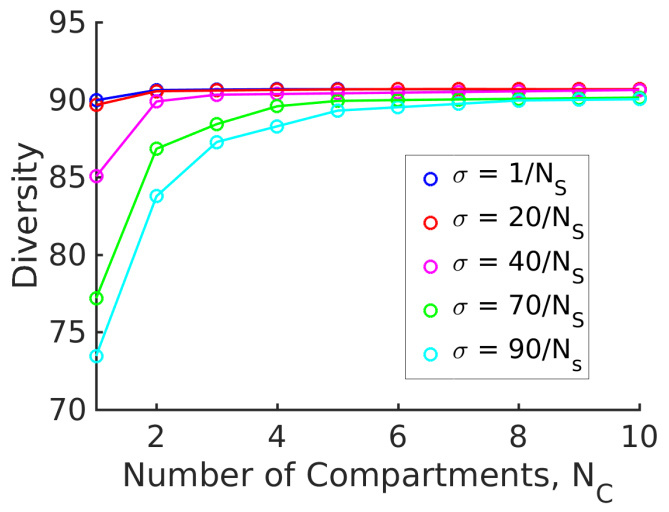

(b)

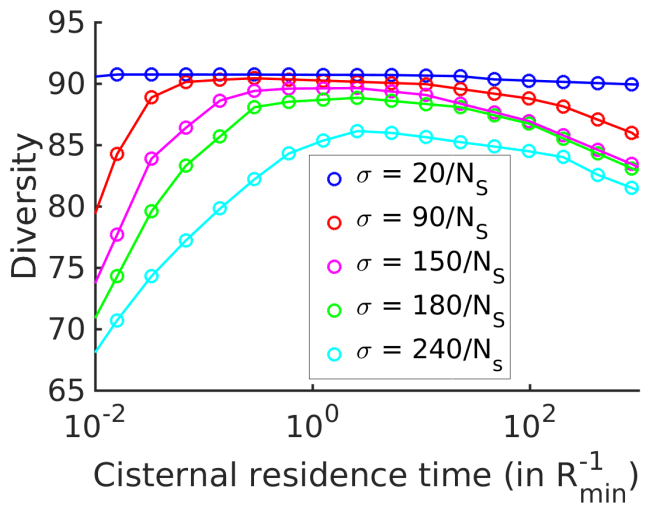

Figure 7. Strategies for achieving high glycan diversity. Diversity versus $N_{C}$ and transport rate $\mu$ at various values of specificity $\sigma$ for fixed $N_{E}=3$. (a) Diversity vs. $N_{C}$ at optimal transport rate $\mu$. Diversity initially increases with $N_{C}$, but eventually levels off. The levelling off starts at a higher $N_{C}$ when $\sigma$ is increased. These curves are bounded by the $\sigma=0$ curve. (b) Diversity vs. cisternal residence time $\left(\mu^{-1}\right)$ in units of the reaction time $\left(R_{\mathrm{min}}^{-1}\right)$ at various value of $\sigma$, for fixed $N_{C}=4$ and $N_{E}=10$.

451 $N_{E}, N_{C}, \sigma:$

where, as before, $\left(\mu_{\max }, \mu_{\min }\right)=(1,0.01) / \mathrm{min}$, and $\left(R_{\max }, R_{\min }\right)=(20,0.018) / \mathrm{min}$, and $c_{t h}=1 / N_{s}$ is the threshold. See Appendix 2 for details on the parameter estimation.

The results displayed in Fig. 7 (a), show that for a fixed specificity $\sigma$, the diversity at first increases with the number of cisternae $N_{C}$, and then saturates at a value that depends on $\sigma$. For very high specificity enzymes, one can achieve very high diversity by appropriately increasing $N_{C}$. This establishes the link between glycan diversity and cisternal number. However, this link is correlational at best, since there are many ways to achieve high glycan diversity - notably by increasing the number of enzymes.

On the other hand, one of the goals of glycoengineering is to produce a particular glycan profile with low heterogeneity (Fisher et al., 2019; Jaiman and Thattai, 2018). For low specificity enzymes, the diversity remains unchanged upon increasing the cisternal residence time. For enzymes with high specificity, the diversity typically shows a non-monotonic variation with the cisternal residence time. At small cisternal residence time, the diversity decreases from the peak because of early exit of incomplete oligomers. At large cisternal residence time the diversity again decreases as more reactions are taken to completion. Note that the peak is generally very flat, this is consistent with the results in (Fisher et al., 2019). To get a sharper peak, as advocated for instance by Vaiman and Thattai, 2018), one might need to increase the number of high specificity enzymes $N_{E}$ further.

\section{Discussion}

The precision of the stereochemistry and enzymatic kinetics of these N-glycosylation reactions (Varki et al., 2009), has inspired a number of mathematical models (Umaña and Bailey, 1997; Krambeck 
et al., 2009; Krambeck and Betenbaugh, 2005) that predict the N-glycan distribution based on the activities and levels of processing enzymes distributed in the Golgi-cisternae of mammalian cells, and compare these predictions with N-glycan mass spectrum data. Models such as the KB2005 model (Umaña and Bailey, 1997; Krambeck and Betenbaugh, 2005; Krambeck et al., 2009) are extremely elaborate (with a network of 22,871 chemical reactions and 7565 oligosaccharide structures) and require many chemical input parameters. These models have an important practical role to play, that of being able to predict the impact of the various chemical parameters on the glycan distribution, and to evaluate appropriate metabolic strategies to recover the original glycoprofile. Additionally, a recent study by Ungar and coworkers (Fisher et al., 2019; Fisher and Ungar, 2016) shows how physical parameters, such as overall Golgi transit time and cisternal number, can be tuned to engineer a homogeneous glycan distribution. Overall, such models can help predict glycosylation patterns and direct glycoengineering projects to optimize glycoform distributions.

Our focus is different. We are interested in the role of glycans as a marker or molecular code of cell identity (Gabius, 2018; Varki, 2017; Pothukuchi et al., 2019), and in particular, understanding enzymatic and transport processes located in the secretory apparatus of the cell that ensure that this code is generated with high fidelity. To do this, we have had to develop a new formal apparatus, that allows us to address these questions and discuss tradeoffs between competing drives. Since our analysis draws on many diverse fields, we provide a short summary of the assumptions, methods and results of the paper before discussing the implications of our work.

(a) The glycan profile on the cell surface is a marker of cell-type identity (Varki et al., 2009; Gabius, 2018; Varki, 2017; Pothukuchi et al., 2019). We define the complexity of a glycan profile to be the minimum number of GMM components required to approximate the profile to within the noise floor. We show that with this definition of complexity, more complex organisms correlate with higher complexity glycan profiles. We use this to analyse the complexity of the glycan profiles of planaria, hydra and mammalian cells (Drickamer and Taylor, 1998).

(b) The glycans at the cell surface are the end product of a sequential chemical processing via a set of enzymes resident in the Golgi cisternae, and transport across cisternae (Varki, 2017, 1998; Pothukuchi et al., 2019). We have proposed a general model for chemical synthesis and transport that, in principle, allows us to compute the synthesized glycan distribution at the cell surface, as a function of the enzymes $N_{E}$, reaction rates $R$, enzyme configurations $L_{r}$ specificity of enzymes $\sigma$, number of cisternae $N_{C}$ and transport rates $\mu$. However the large dimension of the search space makes this optimization intractable. We thus use a simplified synthesis model with fewer parameters; while our quantitative results are based on this simplified model, we believe that at a qualitative level, our results have more general validity.

(c) We define the fidelity of a synthesis mechanism as the minimum normalized Kullback-Leibler (KL) divergence (Cover and Thomas, 2012; MacKay, 2003) between synthesized glycan distribution on the cell surface and a "target" profile.

(d) The results of the optimization over rates $R$ and enzyme configurations $L$ for a given value of $\left(N_{C}, N_{E}, \sigma\right)$ and a target distributions $c^{*}$ of given complexity are Figs. 3-4. Here, we highlight some qualitative consequences of the model:

(i) Keeping the number of enzymes fixed, a more elaborate transport mechanism (via control of $N_{C}$ and $\mu$ ) is essential for synthesizing high complexity target distributions to within a high fidelity, or equivalently, low error (Figs. 4 (a), 4 (b)). Fewer cisternae cannot be compensated for by optimising the enzymatic synthesis via control of parameters $R$, $L$ and $\sigma$. An empirical verification of this would involve a coordinated analysis of the glycan profiles, ultrastructure of golgi and the number of glycosylation enzymes across many species.

(ii) Thus, our study suggests that the requirement that a glycan code of a given complexity be synthesized with sufficiently high fidelity imposes functional control on the Golgi 
cisternal number. It also provides an argument for the evolutionary requirement of multiple-compartments, by demonstrating that the fidelity and robustness of the glycan code arising from a chemical synthesis that involves multiple cisternae is higher than one that involves a single cisterna (keeping everything else fixed) (see Figs. 4(a), 4(b), 6). This feature, that with multiple cisternae and precise enzyme partitioning, one may generically achieve a highly accurate representation of the target distribution, has been highlighted in an algorithmic model of glycan synthesis Jaiman and Thattai (2018).

Combining (i) and (ii), our study quantitatively shows that constructing a high fidelity representation of a complex target distribution, such as those observed in real cells, requires a complex golgi machinery with multiple cisternae, precise enzyme partitioning and control on enzyme specificity. This definition of fidelity of the glycan code, allows us to provide a quantitative argument for the evolutionary requirement of multiplecompartments. While it is possible to produce complex glycan distributions in one compartment using a large number of enzymes, such a design would inevitably require a more elaborate genetic cost.

(iii) Organisms such as plants and algae, have a diverse repertoire of glycans that are utilised in a variety of functions (McFarlane et al., 2014; Koch et al., 2015; O'Neill et al., 2004; Hayashi and Kaida, 2011; Kumar et al., 2011; Gow and Hube, 2012; Atmodjo et al., 2013; Free, 2013; Pauly et al., 2013; Burton and Fincher, 2014). Our study shows that it is optimal to use low specificity enzymes to synthesize target distributions with high diversity (Fig. 7). However, this compromises on the complexity of the glycan distribution, revealing a tension between complexity and diversity. One way of relieving this tension is to have larger $N_{E}$ and $N_{C}$.

(iv) Our study shows that for a fixed $N_{C}$ and $N_{E}$, there is an optimal enzyme specificity that achieves the lowest distance from a given target distribution. As we see in Fig. 4 (d), this optimal enzyme specificity can be very high for highly complex target distributions. Such high specificity can lower fitness when the environment, and hence the target glycan distribution, fluctuates rapidly, and the synthesis parameters cannot change rapidly enough to track the environment (Nam et al., 2012; Peracchi, 2018). This compromise, between robustness to a changing environment and high fidelity in synthesizing high complexity glycan profiles, is achievable by sloppy enzymes coupled with error-correcting mechanisms (Nam et al., 2012; Peracchi, 2018). However, sloppy enzymes create "wrong" glycans, and therefore, ex-post error correcting mechanisms must be in place to correct synthesis errors to ensure high fidelity of the glycan code. A task for the future is to understand the role of intra-cellular transport in providing non-equilibrium proof-reading mechanisms to reduce such coding errors, and its optimal adaptive strategies and plasticity in a time varying environment.

Combining (iii) and (iv), we find that keeping the number of enzymes fixed, having low specificity or sloppy enzymes and larger cisternal number could give rise to a diverse repertoire of functional glycans. Sloppy or promiscuous enzymes bring in the potential for evolvability (Kirschner and Gerhart, 2008), sloppiness allows the system to be stable to random mutations in proteins or variations in the target distribution.

(v) The model solution is degenerate, in the sense that there many equally good global minimas. These degeneracies are both continuous and discrete. The continuous degeneracies corresponds to regions in the reaction rate - transport rate space, moving along which will not change the concentration profile, thus ensuring robustness to internal noise. This suggests that the distribution is robust to slight cell-to-cell variations in these kinetic parameters.

(vi) Our model implies that close to a local minima the inter-cisternal transport rate $\mu$ and 
the specificity of the enzymes $\sigma$ are stiff directions, i.e. the cell should exercise tighter control on $\mu$ and $\sigma$ as compared to the other parameters. The reaction rates close to the local minima are sloppy directions, moving along these directions does not change the glycan profile much.

(e) Taken together, our quantitative analysis of the tradeoffs has deep implications for nonequilibrium self assembly of the Golgi cisternae, and suggests that the nonequilibrium control of cisternal number must involve a coupling of nonequilibrium self assembly of cisternae with enzymatic chemical reaction kinetics (Glick and Malhotra, 1998).

Admittedly the chemical network that we have considered here is much simpler than the chemical network associated with the possible protein modifications in the secretory pathway. For instance, typical N-glycosylation pathways would involve the glycosylation of a variety of GBPs. Further, apart from N-glycosylation, there are other glycoprotein, proteoglycan and glycolipid synthesis pathways (Alberts et al., 2002; Varki et al., 2009; Pothukuchi et al., 2019). Our task has been to to get at a qualitative understanding using quantitative methods and thereby to arrive at general principles. We believe our analysis is generalizable and that the qualitative results we have arrived at would still hold. To conclude, our work establishes the link between the cisternal machinery (chemical and transport) and high fidelity synthesis of a complex glycan code. We find that the pressure to achieve the target glycan code for a given cell type, places strong constraints on the cisternal number and enzyme specificity (Sengupta and Linstedt, 2011). An important implication is that a description of the nonequilibrium self-assembly of a fixed number of Golgi cisternae must combine the dynamics of chemical processing and membrane dynamics involving fission, fusion and transport (Sengupta and Linstedt, 2011; Sachdeva et al., 2016; Sens and Rao, 2013). We believe this is a promising direction for future research.

\section{Acknowledgments}

We thank M. Thattai, A. Jaiman, S. Ramaswamy, A. Varki for discussions, and S. Krishna and R. Bhat for very useful suggestions on the manuscript. We thank our group members at the Simons Centre for many incisive inputs. We are very grateful to P. Babu and P.S. Sabarinath for consultations on the MSMS data and literature. MR acknowledges support from the Department of Atomic Energy (India), under project no. RTI4006, the Simons Foundation (Grant No. 287975), and a J.C. Bose Fellowship from DST-SERB (India). We acknowledge the computational facilities at NCBS. This work has received support under the program Investissements d'Avenir launched by the French Government and implemented by ANR with the references ANR-10-LABX-0038 and ANR-10-IDEX-0001-02 PSL. MR thanks Institut Curie for hosting a visit under the Labex program, and QV thanks the Simons Centre (NCBS) for hosting his visit.

\section{References}

Alberts B, et al. Molecular Biology of the Cell. Garland Science; 2002.

Atmodjo MA, Hao Z, Mohnen D. Evolving views of pectin biosynthesis. Annual review of plant biology. 2013; 64.

Bacharoglou A. Approximation of probability distributions by convex mixtures of gaussian measures. Proceedings of the American Mathematical Society. 2010; 138(7):2619-2628.

Bar-Even A, Milo R, Noor E, Tawfik DS. The moderately efficient enzyme: futile encounters and enzyme floppiness. Biochemistry. 2015; 54(32):4969-4977.

Bard F, Chia J. Cracking the glycome encoder: signaling, trafficking, and glycosylation. Trends in cell biology. 2016; 26(5):379-388. 
Becker B, Melkonian M. The secretory pathway of protists: spatial and functional organization and evolution. Microbiol Mol Biol Rev. 1996; 60(4):697-721.

Berninsone PM, Hirschberg CB. Nucleotide sugar transporters of the Golgi apparatus. Current opinion in structural biology. 2000; 10(5):542-547.

Bialek W. Biophysics: searching for principles. Princeton University Press; 2012.

Blanes S, Casas F, Oteo J, Ros J. The Magnus expansion and some of its applications. Physics Reports. 2009; 470(5-6):151-238

Bonner JT. The origins of multicellularity. Integrative Biology: Issues, News, and Reviews: Published in Association with The Society for Integrative and Comparative Biology. 1998; 1(1):27-36.

Boyd S, Vandenberghe L. Convex optimization. Cambridge university press; 2004.

Burton RA, Fincher GB. Evolution and development of cell walls in cereal grains. Frontiers in plant science. $2014 ; 5: 456$.

Caffaro CE, Hirschberg CB. Nucleotide sugar transporters of the Golgi apparatus: from basic science to diseases. Accounts of chemical research. 2006; 39(11):805-812.

Carroll SB. Chance and necessity: the evolution of morphological complexity and diversity. Nature. 2001; 409(6823):1102-1109.

Casey JR, Grinstein S, Orlowski J. Sensors and regulators of intracellular pH. Nature reviews Molecular cell biology. 2010; 11(1):50.

Changeux JP, Edelstein SJ. Allosteric mechanisms of signal transduction. Science. 2005; 308(5727):1424-1428.

Cover TM, Thomas JA. Elements of information theory. John Wiley \& Sons; 2012.

Cummings RD, Crocker P, Functional Glycomics Database, Consortium for Functional Glycomics; 2020. http: //www.functionalglycomics.org.

Cummings RD, Pierce JM. The challenge and promise of glycomics. Chemistry \& biology. 2014; 21(1):1-15.

D’Angelo G, Capasso S, Sticco L, Russo D. Glycosphingolipids: synthesis and functions. The FEBS journal. 2013; 280(24):6338-6353.

Demetriou M, Granovsky M, Quaggin S, Dennis JW. Negative regulation of T-cell activation and autoimmunity by Mgat5 N-glycosylation. Nature. 2001; 409(6821):733-739.

Dennis JW, Nabi IR, Demetriou M. Metabolism, cell surface organization, and disease. Cell. 2009; 139(7):12291241.

Dmitrieff S, Rao M, Sens P. Quantitative analysis of intra-Golgi transport shows intercisternal exchange for all cargo. Proceedings of the National Academy of Sciences. 2013; 110(39):15692-15697.

Donohoe BS, Kang BH, Staehelin LA. Identification and characterization of COPIa-and COPIb-type vesicle classes associated with plant and algal Golgi. Proceedings of the National Academy of Sciences. 2007; 104(1):163-168.

Drickamer K, Taylor ME. Evolving views of protein glycosylation. Trends in biochemical sciences. 1998; 23(9):321-324.

Du P, Stolovitzky G, Horvatovich P, Bischoff R, Lim J, Suits F. A noise model for mass spectrometry based proteomics. Bioinformatics. 2008; 24(8):1070-1077.

Dwek RA. Glycobiology: toward understanding the function of sugars. Chemical reviews. 1996; 96(2):683-720.

Falk K, Rötzschke O, Stevanovié S, Jung G, Rammensee HG. Allele-specific motifs revealed by sequencing of self-peptides eluted from MHC molecules. Nature. 1991; 351(6324):290.

Fincher CT, Wurtzel O, de Hoog T, Kravarik KM, Reddien PW. Cell type transcriptome atlas for the planarian Schmidtea mediterranea. Science. 2018; 360(6391).

Fisher P, Spencer H, Thomas-Oates J, Wood AJ, Ungar D. Modeling Glycan Processing Reveals Golgi-Enzyme Homeostasis upon Trafficking Defects and Cellular Differentiation. Cell reports. 2019; 27(4):1231-1243. 
Fisher P, Ungar D. Bridging the gap between glycosylation and vesicle traffic. Frontiers in cell and developmental biology. 2016; 4:15.

Free SJ. Fungal cell wall organization and biosynthesis. In: Advances in genetics, vol. 81 Elsevier; 2013.p. 33-82.

Gabius HJ. The sugar code: why glycans are so important. BioSystems. 2018; 164:102-111.

Gagneux P, Varki A. Evolutionary considerations in relating oligosaccharide diversity to biological function. Glycobiology. 1999; 9(8):747-755.

Glick BS, Malhotra V. The curious status of the Golgi apparatus. Cell. 1998; 95(7):883-889.

Gow NA, Hube B. Importance of the Candida albicans cell wall during commensalism and infection. Current opinion in microbiology. 2012; 15(4):406-412.

Gutenkunst RN, Waterfall J, Casey FP, Brown KS, Myers CR, Sethna JP. Universally sloppy parameter sensitivities in systems biology models. PLoS computational biology. 2007; 3(10):e189.

van Halbeek H, Gerwig GJ, Vliegenthart JF, Smits HL, Van Kerkhof PJ, Kramer MF. Terminal $\alpha(1 \diamond 4)$-linked $\mathrm{N}$-acetylglucosamine: A characteristic constituent of doudenal-gland mucous glycoproteins in rat and pig: A high-resolution 1H-NMR study. Biochimica et Biophysica Acta (BBA)-Protein Structure and Molecular Enzymology. 1983; 747(1-2):107-116.

Han X, Zhou Z, Fei L, Sun H, Wang R, Chen Y, Chen H, Wang J, Tang H, Ge W, et al. Construction of a human cell landscape at single-cell level. Nature. 2020; 581(7808):303-309.

Hayashi T, Kaida R. Functions of xyloglucan in plant cells. Molecular Plant. 2011; 4(1):17-24.

Hirschberg CB, Robbins PW, Abeijon C, Transporters of nucleotide sugars, ATP, and nucleotide sulfate in the endoplasmic reticulum and Golgi apparatus. Annual Reviews 4139 El Camino Way, PO Box 10139, Palo Alto, CA 94303-0139, USA; 1998.

Hossler P, Mulukutla BC, Hu WS. Systems analysis of N-glycan processing in mammalian cells. PloS one. 2007; 2(8).

Jaiman A, Thattai M. Algorithmic biosynthesis of eukaryotic glycans. BioRxiv. 2018; p. 440792.

Kellokumpu S. Golgi pH, ion and redox homeostasis: How much do they really matter? Frontiers in cell and developmental biology. 2019; 7:93.

Kirschner MW, Gerhart JC. The plausibility of life. Yale University Press; 2008.

Koch BE, Stougaard J, Spaink HP. Keeping track of the growing number of biological functions of chitin and its interaction partners in biomedical research. Glycobiology. 2015; 25(5):469-482.

Krambeck FJ, Bennun SV, Narang S, Choi S, Yarema KJ, Betenbaugh MJ. A mathematical model to derive $\mathrm{N}$-glycan structures and cellular enzyme activities from mass spectrometric data. Glycobiology. 2009; 19(11):1163-1175.

Krambeck FJ, Betenbaugh MJ. A mathematical model of N-linked glycosylation. Biotechnology and Bioengineering. 2005; 92(6):711-728.

Kumar P, Yang M, Haynes BC, Skowyra ML, Doering TL. Emerging themes in cryptococcal capsule synthesis. Current opinion in structural biology. 2011; 21(5):597-602.

Ladinsky MS, Wu CC, McIntosh S, McIntosh JR, Howell KE. Structure of the Golgi and distribution of reporter molecules at $20 \mathrm{C}$ reveals the complexity of the exit compartments. Molecular biology of the cell. 2002; 13(8):2810-2825.

Llopis J, McCaffery JM, Miyawaki A, Farquhar MG, Tsien RY. Measurement of cytosolic, mitochondrial, and Golgi $\mathrm{pH}$ in single living cells with green fluorescent proteins. Proceedings of the National Academy of Sciences. 1998; 95(12):6803-6808.

Machta BB, Chachra R, Transtrum MK, Sethna JP. Parameter space compression underlies emergent theories and predictive models. Science. 2013; 342(6158):604-607.

MacKay DJ. Information theory, inference and learning algorithms. Cambridge university press; 2003. 
Maverakis E, Kim K, Shimoda M, Gershwin ME, Patel F, Wilken R, Raychaudhuri S, Ruhaak LR, Lebrilla CB. Glycans in the immune system and The Altered Glycan Theory of Autoimmunity: a critical review. Journal of autoimmunity. 2015; 57:1-13.

McFarlane HE, Döring A, Persson S. The cell biology of cellulose synthesis. Annual review of plant biology. $2014 ; 65: 69-94$

Mironov AA, Sesorova IS, Seliverstova EV, Beznoussenko GV. Different Golgi ultrastructure across species and tissues: Implications under functional and pathological conditions, and an attempt at classification. Tissue and Cell. 2017; 49(2):186-201.

Mogelsvang S, Gomez-Ospina N, Soderholm J, Glick BS, Staehelin LA. Tomographic evidence for continuous turnover of Golgi cisternae in Pichia pastoris. Molecular biology of the cell. 2003; 14(6):2277-2291.

Monod J, Wyman J, Changeux JP. On the nature of allosteric transitions: a plausible model. J Mol Biol. 1965; 12(1):88-118.

Moremen KW, Haltiwanger RS. Emerging structural insights into glycosyltransferase-mediated synthesis of glycans. Nature chemical biology. 2019; 15(9):853-864.

Nam H, Lewis NE, Lerman JA, Lee DH, Chang RL, Kim D, Palsson BO. Network context and selection in the evolution to enzyme specificity. Science. 2012; 337(6098):1101-1104.

O'Neill MA, Ishii T, Albersheim P, Darvill AG. Rhamnogalacturonan II: structure and function of a borate crosslinked cell wall pectic polysaccharide. Annu Rev Plant Biol. 2004; 55:109-139.

Owen JA, Punt J, Stranford SA, et al. Kuby immunology. WH Freeman New York; 2013.

Pauly M, Gille S, Liu L, Mansoori N, de Souza A, Schultink A, Xiong G. Hemicellulose biosynthesis. Planta. 2013; 238(4):627-642.

Peracchi A. The Limits of Enzyme Specificity and the Evolution of Metabolism. Trends in biochemical sciences. 2018; .

Pothukuchi P, Agliarulo I, Russo D, Rizzo R, Russo F, Parashuraman S. Translation of genome to glycome: role of the Golgi apparatus. FEBS letters. 2019; 593(17):2390-2411.

Price N, Stevens L. Fundamentals of Enzymology: The cell and molecular biology of catalytic proteins. Oxford University Press; 1999.

Roseman S. Reflections on glycobiology. Journal of Biological Chemistry. 2001; 276(45):41527-41542.

Sachdeva H, Barma M, Rao M. Nonequilibrium description of de novo biogenesis and transport through Golgilike cisternae. Scientific reports. 2016; 6(1):1-10.

Sahadevan S, Antonopoulos A, Haslam SM, Dell A, Ramaswamy S, Babu P. Unique, Polyfucosylated GlycanReceptor Interactions Are Essential for Regeneration of Hydra magnipapillata. ACS chemical biology. 2014; 9(1):147-155.

Savir Y, Tlusty T. Conformational proofreading: the impact of conformational changes on the specificity of molecular recognition. PloS one. 2007; 2(5):e468.

Sengupta AM. Modeling biomolecular networks: an introduction to systems biology. Oxford University Press; 2008.

Sengupta D, Linstedt AD. Control of organelle size: the Golgi complex. Annual review of cell and developmental biology. 2011; 27:57-77.

Sens P, Rao M. (Re) modeling the Golgi. In: Methods in cell biology, vol. 118 Elsevier; 2013.p. 299-310.

Siebert S, Farrell JA, Cazet JF, Abeykoon Y, Primack AS, Schnitzler CE, Juliano CE. Stem cell differentiation trajectories in Hydra resolved at single-cell resolution. Science. 2019; 365(6451).

Stanley P. Golgi glycosylation. Cold Spring Harbor perspectives in biology. 2011; 3(4):a005199.

Stanley P, Sudo T. Microheterogeneity among carbohydrate structures at the cell surface may be important in recognition phenomena. Cell. 1981; 23(3):763-769. 
751 Subramanian SP, Babu P, Palakodeti D, Subramanian R. Identification of multiple isomeric core chitobiose-

752 modified high-mannose and paucimannose N-glycans in the planarian Schmidtea mediterranea. Journal of 753 Biological Chemistry. 2018; 293(18):6707-6720.

754 Trinajstic N. Chemical graph theory. Routledge; 2018.

755 Umaña P, Bailey JE. A mathematical model of N-linked glycoform biosynthesis. Biotechnology and bioengi756 neering. 1997; 55(6):890-908.

757 Varki A, et al. Essentials of Glycobiology. Cold Spring Harbor Laboratory Press; 2009.

758 Varki A. Factors controlling the glycosylation potential of the Golgi apparatus. Trends in cell biology. 1998; 759 8(1):34-40.

Varki A. Evolutionary forces shaping the Golgi glycosylation machinery: why cell surface glycans are universal to living cells. Cold Spring Harbor perspectives in biology. 2011; 3(6):a005462.

762 Varki A. Biological roles of glycans. Glycobiology. 2017; 27(1):3-49.

763 WILLS C, GREEN DR. A genetic herd-immunity model for the maintenance of MHC polymorphism. Immunolog764 ical reviews. 1995; 143(1):263-292.

765 Winterburn P, Phelps C. The significance of glycosylated proteins. Nature. 1972; 236(5343):147-151.

766 Yang M, Fehl C, Lees KV, Lim EK, Offen WA, Davies GJ, Bowles DJ, Davidson MG, Roberts SJ, Davis BG. Func767 tional and informatics analysis enables glycosyltransferase activity prediction. Nature chemical biology. 2018; $768 \quad 14(12): 1109-1117$.

Yuan M, Lin Y. Model selection and estimation in regression with grouped variables. Journal of the Royal Statistical Society: Series B (Statistical Methodology). 2006; 68(1):49-67. 


\section{Appendix 1}

\section{Constructing target distributions for glycans of a given cell type}

The distribution of the glycans on the cell surface is obtained via mass spectrometry. The $x$ axis of mass spectroscopy (MS) graphs is mass/charge of the ionised sample molecules and the $y$-axis is relative intensity corresponding to each mass/charge value, taking the highest intensity as $100 \%$. This relative intensity roughly correlates with the relative abundances of the molecules in the sample.

The raw MS data is noisy and cannot be directly used as the target distribution in our optimization problem. There are three major sources of noise in the MS data (Du et al., 2008): chemical noise in the sample, the Poisson noise associated with detecting discrete events, and the Nyquist-Johnson noise associated with any charge system. We propose a simple model that accounts for the chemical noise and the Poisson sampling noise. Using this noise model and the available MS data, we generate parametric bootstrap samples of glycan measurements, and fit a Gaussian Mixture Model (GMM) on this sample to approximate the glycan distribution. This GMM probability distribution is used as the target distribution in our numerical experiments.

The MS data obtained from (Cummings and Crocker, 2020; Subramanian et al., 2018; Sahadevan et al., 2014) had mass ranging between 500 to 5000 Daltons with intensity reported at every 0.0153 Daltons. We first bin this MS data into 180 bins and take the maximum value within each bin as the value of intensity for that bin. Fig. 1 plots the raw MS data and the binned distribution. Next, we describe the parametric bootstrap model that we used to generate the glycan data. Let $\bar{I}_{k}$ represents the relative intensity of the $k$-th bin in the binned MS graph. We generate a sample population of glycans using the MS data in the following way:

1. Poisson sampling noise: The MS data does not have absolute count information. We assume an arbitrary maximum count $I_{\max }$, and define the intensity $I_{k}=I_{\max } \bar{I}_{k}$. The plots in Appendix 1 Fig. 2 (a) show that the results are not sensitive to the specific value of $I_{\max }$.

2. Chemical noise: The sample used for MS analysis also contains small amounts of molecules that are not glycans. These appear as the very small peaks in the MS data. We assume that the probability $p_{k}$ that the peak at index $k$ corresponds to a glycan is given by

$$
p_{k}=1-e^{-\frac{I_{k}}{I_{\max }}}=1-e^{-\bar{I}_{k}}
$$

which adequately suppresses this chemical noise.

3. Bootstrapped glycan data: The count $n_{k}$ at the glycan index $k$ is distributed according to the following distribution:

$$
n_{k}=\left\{\begin{array}{lll}
0 & \left(1-p_{k}\right) & n=0 \\
n & p_{k} e^{-I_{k}} \frac{\left.I_{k}\right)^{n}}{n !} & n \geq 1 .
\end{array}\right.
$$

We assume that the MS data was generated from $N$ different cells. Thus, the total count at glycan index $k$ is given by the sum of $N$ i.i.d. samples distributed according to the distribution above. We in Appendix 1 Fig. 2 (b) show that results are insensitive to $N$. We normalize the count distribution by the total number of counts across all the bins to obtain the bootstrapped probability mass function $p_{T}$. 
The bootstrapped distribution $p_{T}$ is is noisy, and hence can not be used directly as the target distribution. We use a Gaussian Mixture Model (GMM) based approach to de-noise the raw data. The advantage of using a GMM based approach is that it creates an easily interpretable hierarchy of increasingly more detailed distributions to approximate the mass spectrometry profile. We define the complexity of a mass spectrometry profile as the minimum number of components (individual Gaussians) in the GMM model required to approximate it. The details of the GMM calculations are as follows. We fix the number of components $m$. We want to approximate the bootstrapped probability $p_{T}$ by the $m$-component mixture of Gaussian distributions $p_{G M M}(\theta)=\sum_{i=1}^{m} w_{i} \mathcal{N}_{\eta_{i}, \Delta_{i}}$, where $\mathcal{N}_{\eta_{i}, \Delta_{i}}$ denotes the Gaussian distribution with mean $\eta_{i}$ and variance $\Delta_{i}, w_{i} \geq 0$ and $\sum_{i=1}^{m} w_{i}=1$, the parameter vector $\boldsymbol{\theta}=(\boldsymbol{w}, \boldsymbol{\eta}, \boldsymbol{\Delta})$ We compute the optimal $m$-component GMM approximation by minimizing the KL-divergence $D\left(p_{T} \| p_{G M M}(\boldsymbol{\theta})\right)$ as a function of parameter vector $\boldsymbol{\theta}$. Since

$$
\begin{aligned}
D\left(p_{T} \| p_{G M M}(\theta)\right) & :=\sum_{k=1}^{N_{s}} p_{T}(k) \log \left(\frac{p_{T}(k)}{\sum_{i}^{m} w_{i} \mathcal{N}_{\eta_{i}, \Delta_{i}}(k)}\right) \\
& =\sum_{k=1}^{N_{s}} p_{T}(k) \log p_{T}(k)-\sum_{k=1}^{N_{s}} p_{T}(k) \log \left(\sum_{i}^{m} w_{i} \mathcal{N}_{\eta_{i}, \Delta_{i}}(k)\right),
\end{aligned}
$$

the optimization problem $\min _{\theta} D\left(p_{T} \| p_{G M M}(\theta)\right)$ is equivalent to

$$
\max _{\boldsymbol{\theta}} g(\boldsymbol{\theta}):=\sum_{k=1}^{N_{S}} p_{T}(k) \log \left(\sum_{i=1}^{m} w_{i} \mathcal{N}_{\eta_{i}, \Delta_{i}}(k)\right)
$$

This is a non-convex optimization. We use an Expectation-Maximization (EM) based iterative heuristic to compute a local maximum. Let $\boldsymbol{\theta}^{(t)}$ denote the current value of the parameters. For each component $i=1, \ldots, m$, and index $k=1, \ldots, N_{s^{\prime}}$ define

$$
z_{i}^{(t)}(k)=\frac{w_{i}^{(t)} \mathcal{N}_{\eta_{i}^{(t)}, \Delta_{i}^{(t)}}(k)}{\sum_{j=1}^{m} w_{j}^{(t)} \mathcal{N}_{\eta_{j}^{(t)}, \Delta_{j}^{(t)}}(k)} .
$$

Then $z_{i}^{(t)}(k) \geq 0$, and $\sum_{i=1}^{m} z_{i}^{(t)}(k)=1$. We interpret $z_{i}^{(t)}(k)$ as the probability that the count in bin $k$ came from component $i$. Define

$$
Q\left(\boldsymbol{\theta}, \boldsymbol{\theta}^{(t)}\right)=\sum_{k=1}^{N_{S}} \sum_{i=1}^{m} p_{T}(k) z_{i}^{(t)}(k) \log \left(\frac{w_{i} \mathcal{N}_{\eta_{i}, \Delta_{i}}(k)}{z_{i}^{(t)}(k)}\right)
$$

Then we have that

$$
Q(\hat{\boldsymbol{\theta}}, \hat{\boldsymbol{\theta}})=\sum_{k=1}^{N_{S}} \sum_{i=1}^{m} p_{T}(k) \hat{z}_{i}(k) \log \left(\sum_{i=1}^{m} w_{i} \mathcal{N}_{\eta_{i}, \Delta_{i}}(k)\right)=\sum_{k=1}^{N_{S}} p_{T}(k) \log \left(\sum_{i=1}^{m} w_{i} \mathcal{N}_{\eta_{i}, \Delta_{i}}(k)\right)=g(\hat{\boldsymbol{\theta}}),
$$

and

$$
g(\boldsymbol{\theta})=\sum_{k=1}^{N_{S}} p_{T}(k) \log \left(\sum_{i=1}^{m} \frac{w_{i} \mathcal{N}_{\eta_{i}, \Delta_{i}}(k)}{z_{i}^{(t)}(k)} z_{i}^{(t)}(k)\right) \geq \sum_{k=1}^{N_{S}} \sum_{i=1}^{m} p_{T}(k) z_{i}^{(t)}(k) \log \left(\frac{w_{i} \mathcal{N}_{\eta_{i}, \Delta_{i}}(k)}{z_{i}^{(t)}(k)}\right)=Q\left(\boldsymbol{\theta}, \boldsymbol{\theta}^{(t)}\right) .
$$

Define

$$
\theta^{(t+1)}=\arg \max _{\theta} Q(\theta, \hat{\theta})
$$

Then, we have that

$$
g\left(\theta^{(t+1)}\right) \geq Q\left(\theta^{(t+1)}, \theta^{(t)}\right) \geq Q\left(\theta^{(t)}, \theta^{(t)}\right)=g\left(\theta^{(t)}\right) .
$$

Therefore, the iterative algorithm in (8) generates a sequence $\left\{\theta^{(t)}: t \geq 1\right\}$ with non-decreasing values of $g$, and the sequence converges to a local maximum. Next, we show that the optimization in (8) can be computed efficiently. 


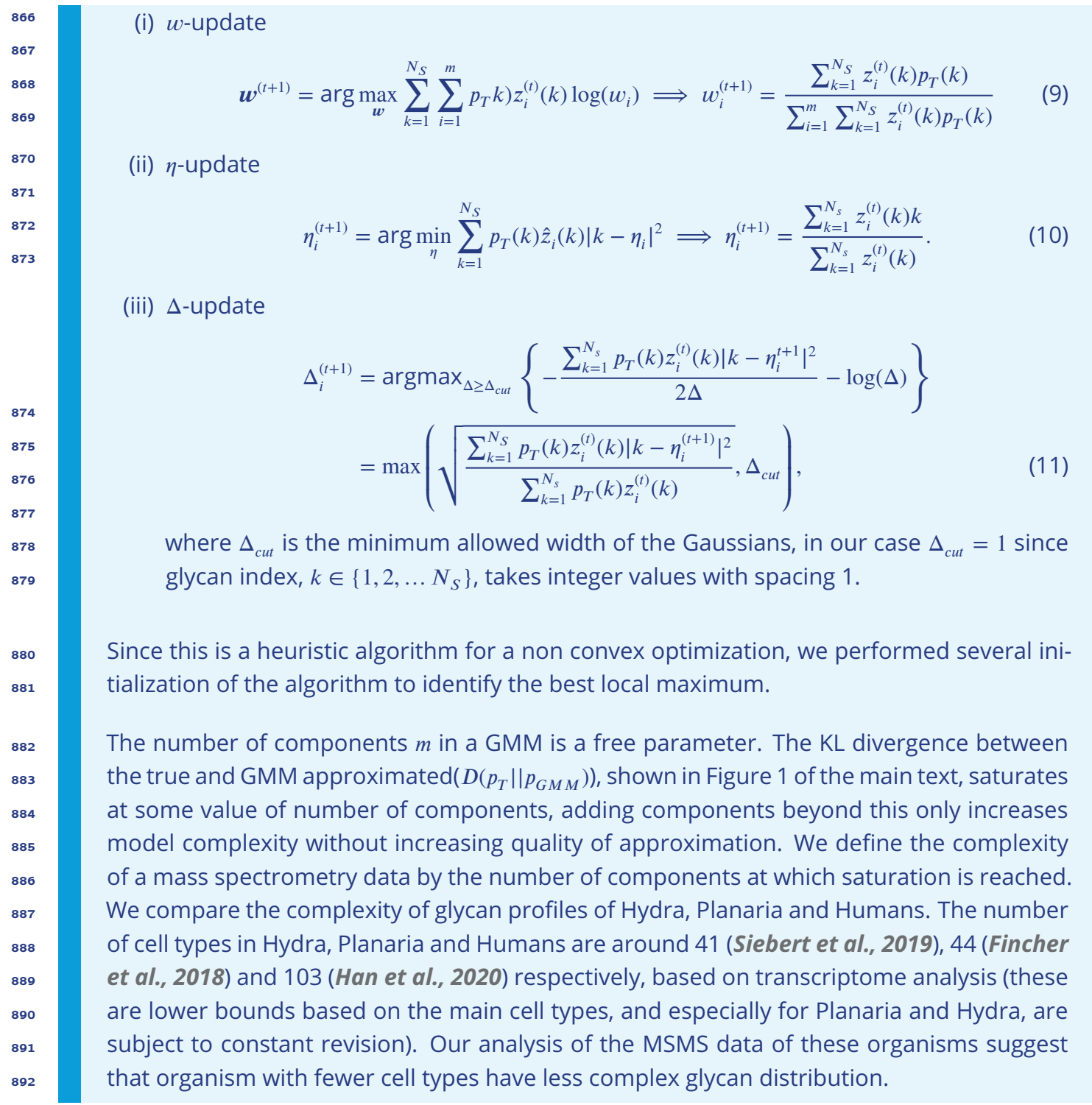




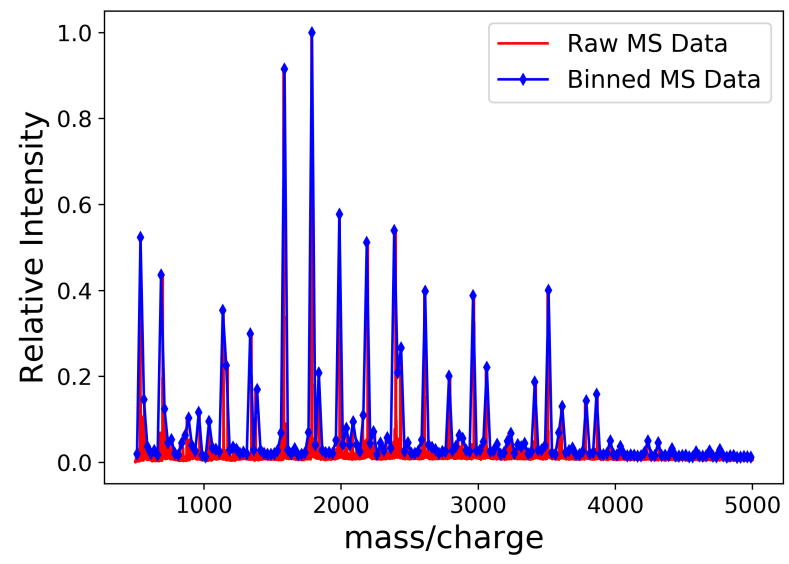

Figure 1. The binned MS data (blue) approximates the raw MS data (red) very well. We use this binned data for GMM approximation of the MS data.

(a)

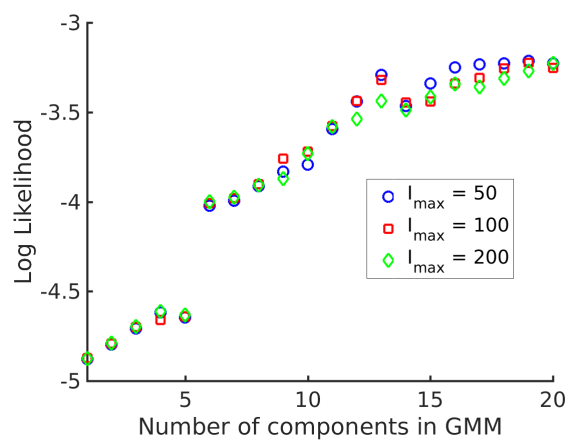

(b)

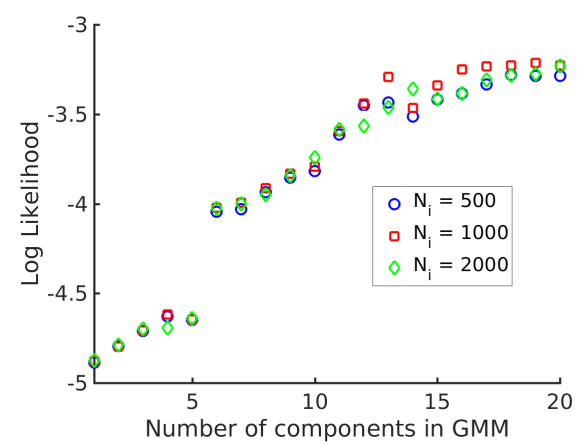

Figure 2. Log likelihood vs. number of components $(N)$ in the GMM. We see that the log likelihood saturates at around $N=20$, thus 20-GMM is a very good representation of the MS-data from human T-cells. The different symbols are for (a) different values of the maximum intensity $I_{\max }=50,100,200$ and (b) different values of the number of i.i.d samples $N_{i}=500,1000,2000$, showing the insensitivity of the log likelihood to the value of $I_{\max }$ and $N_{i}$. 


\section{Appendix 2}

\section{Parameter estimation}

The typical transport time of glycoproteins across the Golgi complex is estimated to be in the range 15-20 mins. (Umaña and Bailey, 1997), which corresponds to the transport rate $\mu=0.18 / \mathrm{min}$. We bound the transport rate for our optimization between $0.01 / \mathrm{min}$ and $1 / \mathrm{min}$.

Next, we estimate the range of values for the chemical reaction rates. The injection rate $q$ is in the range $100-1500 \mathrm{pmol} / 10^{6}$ cell $24 \mathrm{~h}$ (Umaña and Bailey, 1997; Krambeck et al., 2009). For our calculation we set $q=387.30 \mathrm{pmol} / 10^{6}$ cells $24 \mathrm{hr}=0.27 \mathrm{pmol} / 10^{6}$ cells min, where 387.30 is the geometric mean of 100 and 1500 . We set the range for the enzymatic rate $R$ to be

$$
R_{\min }=\min _{\alpha}\left\{\frac{V_{\max }^{(\alpha)} / v}{K_{M}^{(\alpha)}+\frac{1}{v} \frac{q}{\mu}}\right\} \leq R \leq R_{\max }=\max _{\alpha}\left\{\frac{V_{\max }^{(\alpha)} / v}{K_{M}^{(\alpha)}}\right\} .
$$

where $K_{M}^{(\alpha)}$ and $V_{\max }^{(\alpha)}$ denote the Michaelis constants and $V_{\max }$ of the $\alpha$-th enzyme. The conversion from 1 pmoles $/ 10^{6}$ cells to concentration can be obtained by taking cisternal volume $(v)$ to be $2.5 \mu m^{3}$ (Umaña and Bailey, 1997; Krambeck et al., 2009). This gives

$$
1 \text { pmoles } / 10^{6} \text { cells }=\frac{10^{-12} \text { moles }}{10^{6} \times 2.5 \times 10^{-18} \times 10^{3} \text { litre }}=400 \mu M .
$$

In Table 1 we report the parameters for the 8 enzymes taken from Table 3 in Umaña and Bailey (1997). From these parameters it follows that

$$
\begin{aligned}
R_{\min } & =\min _{\alpha}\left\{\frac{V_{\max }^{(\alpha)} / v}{K_{M}^{(\alpha)}+\frac{1}{v} \frac{q}{\mu}}\right\} \\
& =\frac{V_{\max }^{(7)} / v}{K_{M}^{(7)}+\frac{1}{v} \frac{q}{\mu}}=\frac{.16 \times 400 \mu M / \mathrm{min}}{3400 \mu M+149.4 \mu M}=0.018 \mathrm{~min}^{-1} \\
R_{\max } & =\max _{\alpha}\left\{\frac{V_{\max }^{(\alpha)} / v}{K_{M}^{(\alpha)}}\right\} \\
& =\frac{V_{\max }^{(1)} / v}{K_{M}^{(1)}}=\frac{5 \times 400 \mu M / \mathrm{min}}{100 \mu M}=20 \mathrm{~min}^{-1}
\end{aligned}
$$

\begin{tabular}{|c|c|c|}
\hline$\alpha$ & $\begin{array}{c}K_{M}^{(\alpha)} \\
(\mu \mathrm{mol})\end{array}$ & $\begin{array}{c}V_{\max }^{(\alpha)} \\
\left(\mathrm{pmol} / 10^{6} \text { cell-min) }\right.\end{array}$ \\
\hline \hline 1 & 100 & 5 \\
2 & 260 & 7.5 \\
3 & 200 & 5 \\
4 & 100 & 5 \\
5 & 190 & 2.33 \\
6 & 130 & .16 \\
7 & 3400 & .16 \\
8 & 4000 & 9.66 \\
\hline
\end{tabular}

Table 1. Enzyme parameters taken from Table 3 in Umaña and Bailey (1997) that we use to calculate the bounds on the reaction rate $R$. Here $K_{M}^{(\alpha)}$ and $V_{\max }^{(\alpha)}$ denote the Michaelis constant and $V_{\max }$ of the $\alpha$-th enzyme. 


\section{Appendix 3}

\section{Kinetics of sequential chemical reactions and transport}

On including the rates of injection $(q)$ and inter-cisternal transport $\mu^{(j)}$ from the cisterna $j$ to $j+1$, into the chemical reaction kinetics, the change in substrate concentrations $c_{k}^{(j)}$ with time is given by

$$
\begin{aligned}
\frac{d c_{1}^{(1)}}{d t}= & q-\sum_{\alpha=1}^{N_{E}} \frac{V(1,1, \alpha) P^{(1)}(1, \alpha) c_{1}^{(1)}}{M(1,1, \alpha)\left(1+\sum_{k^{\prime}=1}^{N_{s}} \frac{P(1)\left(k^{\prime}, \alpha\right) c_{k^{\prime}}^{(1)}}{M\left(1, k^{\prime}, \alpha\right)}\right.}-\mu^{(1)} c_{1}^{(1)} \\
\frac{d c_{k}^{(1)}}{d t}= & \sum_{\alpha=1}^{N_{E}} \frac{V(1, k-1, \alpha) P^{(1)}(k-1, \alpha) c_{k-1}^{(1)}}{M(1, k-1, \alpha)\left(1+\sum_{k^{\prime}=1}^{N_{s}} \frac{P(1)\left(k^{\prime}, \alpha\right) c_{k^{\prime}}^{(1)}}{M\left(1, k^{\prime}, \alpha\right)}\right)} \\
& -\sum_{\alpha=1}^{N_{E}} \frac{V(1, k, \alpha) P^{(1)}(k, \alpha) c_{k}^{(1)}}{M(1, k, \alpha)\left(1+\sum_{k^{\prime}=1}^{N_{s}} \frac{P^{(1)}\left(k^{\prime}, \alpha\right) c_{k^{\prime}}^{(1)}}{M\left(1, k^{\prime}, \alpha\right)}\right)}-\mu^{(1)} c_{k}^{(1)} \\
\frac{d c_{N_{s}}^{(1)}}{d t}= & \sum_{\alpha=1}^{N_{E}} \frac{V\left(1, N_{s}-1, \alpha\right) P^{(1)}\left(N_{s}-1, \alpha\right) c_{N_{s}-1}^{(1)}}{M\left(1, N_{s}-1, \alpha\right)\left(1+\sum_{k^{\prime}=1}^{N_{s}} \frac{P^{(1)}\left(k^{\prime}, \alpha c_{k^{\prime}}\right.}{M\left(1, k^{\prime}, \alpha\right)}\right.}-\mu^{(1)} c_{N_{s}}^{(1)}
\end{aligned}
$$

for cisterna-1, and

$$
\begin{aligned}
\frac{d c_{1}^{(j)}}{d t}= & \mu^{(j-1)} c_{1}^{(j-1)}-\sum_{\alpha=1}^{N_{E}} \frac{V(j, 1, \alpha) P^{(j)}(1, \alpha) c_{1}^{(j)}}{M(j, 1, \alpha)\left(1+\sum_{k^{\prime}=1}^{N_{s}} \frac{P(j)\left(k^{\prime}, \alpha\right) c_{k^{\prime}}^{(j)}}{M\left(j, k^{\prime}, \alpha\right)}\right.}-\mu^{(j)} c_{1}^{(j)} \\
\frac{d c_{k}^{(j)}}{d t}= & \mu^{(j-1)} c_{k}^{(j-1)}+\sum_{\alpha=1}^{N_{E}} \frac{V(j, k-1, \alpha) P^{(j)}(k-1, \alpha) c_{k-1}^{(j)}}{M(j, k-1, \alpha)\left(1+\sum_{k^{\prime}=1}^{N_{s}} \frac{P(j)\left(k^{\prime}, \alpha\right) c^{\prime}}{M\left(j, k^{\prime}, \alpha\right)}\right)} \\
& -\sum_{\alpha=1}^{N_{E}} \frac{V(j, k, \alpha) P^{(j)}(k, \alpha) c_{k}^{(j)}}{M(j, k, \alpha)\left(1+\sum_{k^{\prime}=1}^{N_{s}} \frac{P(j)\left(k^{\prime}, \alpha\right) c_{k^{\prime}}^{(j)}}{M\left(j, k^{\prime}, \alpha\right)}\right)}-\mu^{(j)} c_{k}^{(j)} \\
\frac{d c_{N_{s}}^{(j)}}{d t}= & \mu^{(j-1)} c_{N_{s}}^{(j-1)}+\sum_{\alpha=1}^{N_{E}} \frac{V\left(j, N_{s}-1, \alpha\right) P^{(j)}\left(N_{s}-1, \alpha\right) c_{N_{s}-1}^{(j)}}{M\left(j, N_{s}-1, \alpha\right)\left(1+\sum_{k^{\prime}=1}^{N_{s}} \frac{P(j)\left(k^{\prime}, \alpha c_{k^{\prime}}^{(j)}\right.}{M\left(j, k^{\prime}, \alpha\right)}\right.}-\mu^{(j)} c_{N_{s}}^{(j)}
\end{aligned}
$$

for cisternae $j=2,3, \ldots, N_{C}$. These set of dynamical equations (13)-(14), with initial conditions, can be solved to obtain the concentration $c_{k}^{(j)}(t)$ for $t \geq 0$. Equations (13)-(14) automatically obey the conservation law for the protein concentration $(p)$, i.e., the total protein concentration $p^{(j)}=\sum_{k^{\prime}=1}^{N_{s}} c_{k^{\prime}}^{(j)}$ in the $j$-th cisterna automatically satisfies,

$$
\begin{aligned}
\frac{d p^{(1)}}{d t} & =q-\mu^{(1)} p^{(1)} \\
\frac{d p^{(j)}}{d t} & =\mu^{(j-1)} p^{(j-1)}-\mu^{(j)} p^{(j)}
\end{aligned}
$$

for $j=2,3, \ldots N_{C}$.

At steady state, the left hand side of in equations (13)-(14) is set to zero, which after rescaling the kinetic parameters in terms of the injection rate $q$, i.e. $V(j, k, \alpha)=V(j, k, \alpha) / q$ and $\mu^{(j)}=\mu^{(j)} / q$, gives the following recursion relations for the steady state concentrations of 
the glycans in each cisterna. In the first cisterna,

$$
\begin{aligned}
& c_{1}^{(1)}=\frac{1}{\mu^{(1)}+\sum_{\alpha=1}^{N_{E}} \frac{V(1,1, \alpha) P^{(1)}(1, \alpha) c_{1}^{(1)}}{M(1,1, \alpha)\left(1+\sum_{k^{\prime}=1}^{N_{s}} \frac{P^{(1)}\left(k^{\prime}, \alpha\right) c_{k^{\prime}}^{(1)}}{M\left(1, k^{\prime}, \alpha\right)}\right)}} \\
& \frac{\sum_{\alpha=1}^{N_{E}} \frac{V(1, k-1, \alpha) P^{(1)}(k-1, \alpha) c_{k-1}^{(1)}}{M(1, k-1, \alpha)\left(1+\sum_{k^{\prime}=1}^{N_{s}} \frac{P^{(1)}\left(k^{\prime}, \alpha\right) c_{k^{\prime}}^{(1)}}{M\left(1, k^{\prime}, \alpha\right)}\right)}}{\mu^{(1)}+\sum_{\alpha=1}^{N_{E}} \frac{V(1, k, \alpha) P^{(1)}(k, \alpha) c_{k}^{(1)}}{M(1, k, \alpha)\left(1+\sum_{k^{\prime}=1}^{N_{s}} \frac{P^{(1)}\left(k^{\prime}, \alpha\right) c_{k^{\prime}}^{(1)}}{M\left(1, k^{\prime}, \alpha\right)}\right)}} \\
& \frac{\sum_{\alpha=1}^{N_{E}} \frac{V\left(1, N_{s}-1, \alpha\right) P^{(1)}\left(N_{s}-1, \alpha\right) c_{N_{s}-1}^{(1)}}{M\left(1, N_{s}-1, \alpha\right)\left(1+\sum_{k^{\prime}=1}^{N_{s}} \frac{P^{(1)}\left(k^{\prime}, \alpha\right) c_{k^{\prime}}^{(1)}}{M\left(1, k^{\prime}, \alpha\right)}\right)}}{\mu^{(1)}}
\end{aligned}
$$

and in cisternae $j \geq 2$,

$$
\begin{aligned}
c_{1}^{(j)}= & \frac{\mu^{(j-1)} c_{1}^{(j-1)}}{\mu^{(j)}+\sum_{\alpha=1}^{N_{E}} \frac{V(j, 1, \alpha) P^{(j)}(1, \alpha) c_{1}^{(j)}}{M(j, 1, \alpha)\left(1+\sum_{k^{\prime}=1}^{N_{s}} \frac{P^{(j)}\left(k^{\prime}, \alpha\right) c_{k^{\prime}}^{(j)}}{M\left(j, k^{\prime}, \alpha\right)}\right)}} \\
c_{k}^{(j)}= & \frac{\mu^{(j-1)} c_{k}^{(j-1)}+\sum_{\alpha=1}^{N_{E}} \frac{V(j, k-1, \alpha) P^{(j)}(k-1, \alpha) c_{k-1}^{(j)}}{M(j, k-1, \alpha)\left(1+\sum_{k^{\prime}=1}^{N_{s}} \frac{P^{(j)}\left(k^{\prime}, \alpha\right) c_{k^{\prime}}^{(j)}}{M\left(j, k^{\prime}, \alpha\right)}\right)}}{\mu^{(j)}+\sum_{\alpha=1}^{N_{E}} \frac{V(j, k, \alpha) P^{(j)}(k, \alpha) c_{k}^{(j)}}{M(j, k, \alpha)\left(1+\sum_{k^{\prime}=1}^{N_{s}} \frac{P^{(j)}\left(k^{\prime}, \alpha\right) c_{k^{\prime}}^{(j)}}{M\left(j, k^{\prime}, \alpha\right)}\right)}} \\
c_{N_{s}}^{(j)}= & \frac{\mu^{(j-1)} c_{N_{s}}^{(j-1)}+\sum_{\alpha=1}^{N_{E}} \frac{V\left(j, N_{s}-1, \alpha\right) P^{(j)}\left(N_{s}-1, \alpha\right) c_{N_{s}-1}^{(j)}}{M\left(j, N_{s}-1, \alpha\right)\left(1+\sum_{k^{\prime}=1}^{N_{s}} \frac{P^{(j)} \frac{\left.\left.k^{\prime}, \alpha\right) c^{\prime}\right)}{M\left(j, k^{\prime}, \alpha\right)}}{M^{(j)}}\right)}}{\mu^{(j)}}
\end{aligned}
$$

Equations (15)-(16) automatically imply that the total steady state glycan concentration in each cisterna $j=1, \ldots, N_{c}$ is given by

$$
\sum_{k=1}^{N_{s}} c_{k}^{(j)}=\frac{1}{\mu^{(j)}} .
$$




\section{Reformulation of Optimization A}

Define a new set of parameters,

$$
R(j, k, \alpha)=\sum_{\alpha=1}^{N_{E}} \frac{V(j, k, \alpha)}{M(j, k, \alpha)\left(1+\sum_{k^{\prime}=1}^{N_{s}} \frac{P(j)\left(k^{\prime}, \alpha\right)\left(c_{k^{\prime}}^{(j)}\right.}{M\left(j, k^{\prime}, \alpha\right)}\right)}
$$

where $\mathbf{c}$ denotes the steady state glycan concentration corresponding to a specific ( $\boldsymbol{\mu}, \mathbf{M}, \mathbf{V}, \mathbf{L})$. Define $\mathbf{v}$ by the following set of linear equations:

$$
\begin{aligned}
& v_{1}^{(1)}=\frac{1}{\mu^{(1)}+\sum_{\alpha=1}^{N_{E}} R(1,1, \alpha) P^{(1)}(1, \alpha)} \\
& v_{k}^{(1)}=\frac{v_{k-1}^{(1)} \sum_{\alpha=1}^{N_{E}} R(1, k-1, \alpha) P^{(1)}(k-1, \alpha)}{\mu^{(1)}+\sum_{\alpha=1}^{N_{E}} R(1, k, \alpha) P^{(1)}(k, \alpha)} \\
& v_{N_{s}}^{(1)}=\frac{v_{N_{s}-1}^{(1)} \sum_{\alpha=1}^{N_{E}} R\left(1, N_{s}-1, \alpha\right) P^{(1)}\left(N_{s}-1, \alpha\right)}{\mu^{(1)}}
\end{aligned}
$$

for $j=1$, and

$$
\begin{aligned}
v_{1}^{(j)}= & \frac{v_{1}^{(j-1)} \mu^{(j-1)}}{\mu^{(j)}+\sum_{\alpha=1}^{N_{E}} R(j, 1, \alpha) P^{(j)}(1, \alpha)} \\
v_{k}^{(j)}= & \frac{v_{k}^{(j-1)} \mu^{(j-1)}}{\mu^{(j)}+\sum_{\alpha=1}^{N_{E}} R(j, k, \alpha) P^{(j)}(k, \alpha)} \\
& +\frac{v_{k-1}^{(j)} \sum_{\alpha=1}^{N_{E}} R(j, k-1, \alpha) P^{(j)}(k-1, \alpha)}{\mu^{(j)}+\sum_{\alpha=1}^{N_{E}} R(j, k, \alpha) P^{(j)}(k, \alpha)} \\
v_{N_{s}}^{(j)}= & \frac{v_{N_{s}}^{(j-1)} \sum_{\alpha=1}^{N_{E}} R\left(j, N_{s}-1, \alpha\right) P^{(j)}\left(N_{s}-1, \alpha\right)}{\mu^{(j)}}+\frac{v_{N_{s}}^{(j-1)} \mu^{(j-1)}}{\mu^{(j)}}
\end{aligned}
$$

for $j=2, \ldots, N_{C}$. Then, by the definition of $\mathbf{R}$ in (17), it trivially follows that the steady state concentration c corresponding to $(\boldsymbol{\mu}, \mathbf{M}, \mathbf{V}, \mathbf{L})$ is a solution for (18)-(19).

Next, we show that for $\mathbf{v}$ obtained from (18)-(19) for any parameter $(\boldsymbol{\mu}, \mathbf{R}, \mathbf{L})$, there exists parameter $(\boldsymbol{\mu}, \mathbf{M}, \mathbf{V}, \mathbf{L})$ such that (15)-(16) are automatically satisfied when we set $\mathbf{c}=\mathbf{v}$, i.e. $\mathbf{v}$ is the steady state concentration for $(\boldsymbol{\mu}, \mathbf{M}, \mathbf{V}, \mathbf{L})$, and vice versa. Let

$$
\mathcal{A}=\left\{\begin{array}{ll}
{\left[c_{k}^{(j)}\right]_{j, k}: \begin{array}{l}
\mu^{(j)} \geq 0, M(j, k, \alpha) \geq 0, V(j, k, \alpha) \geq 0, l_{\alpha}^{(j)} \geq 0, \\
{\left[c_{k}^{(j)}\right]_{j k} \text { given by }(15) \text { and }(16),}
\end{array}}
\end{array}\right\}
$$

and let

$$
B=\left\{\left[v_{k}^{(j)}\right]_{j, k}: \begin{array}{l}
\mu^{(j)} \geq 0, R(j, k, \alpha) \geq 0, l_{\alpha}^{(j)} \geq 0 \\
{\left[v_{k}^{(j)}\right]_{j, k} \text { given by }(18) \text { and }(19)}
\end{array}\right\} .
$$


Then, our task is to show that $\mathcal{A}=\mathcal{B}$. Suppose $\left[c_{k}^{(j)}\right]_{j, k} \in \mathcal{A}$. Let $[M(j, k, \alpha)],[V(j, k, \alpha)]$ and $\left[l_{\alpha}^{(j)}\right]$ be the corresponding parameters. Define

$$
R(j, k, \alpha)=\sum_{\alpha=1}^{N_{E}} \frac{V(j, k, \alpha)}{M(j, k, \alpha)\left(1+\sum_{k^{\prime}=1}^{N_{s}} \frac{P^{(j)}\left(k^{\prime}, \alpha\right) c_{k^{\prime}}^{(j)}}{M\left(j, k^{\prime}, \alpha\right)}\right)} \geq 0
$$

Then $\left[c_{k}^{(j)}\right]_{j, k} \in \mathcal{B}$.

Next, suppose $\left[v_{k}^{(j)}\right]_{j, k} \in \mathcal{B}$. Let $[R(j, k, \alpha)],\left[l_{\alpha}^{(j)}\right]$ denote the corresponding parameters. Since $\sum_{k=1}^{N_{s}} v_{k}^{(j)}=1 / \mu^{(j)}<\infty$, it follows that $\sum_{k=1}^{N_{s}} P^{(j)}(k, \alpha) v_{k}^{(j)}<1 / \mu^{(j)}<\infty$. Thus, there exists parameters $[M(j, k, \alpha)],[V(j, k, \alpha)]$ and $\left[l_{\alpha}^{(j)}\right]$ such that

$$
R(j, k, \alpha)=\sum_{\alpha=1}^{N_{E}} \frac{V(j, k, \alpha)}{M(j, k, \alpha)\left(1+\sum_{k^{\prime}=1}^{N_{s}} \frac{P(j)\left(k^{\prime}, \alpha v_{k^{\prime}}^{(j)}\right.}{M\left(j, k^{\prime}, \alpha\right)}\right)}
$$

Therefore, $\left[v_{k}^{(j)}\right]_{j, k}$ satisfy (15) and (16), i.e. $\left[v_{k}^{(j)}\right]_{j, k} \in \mathcal{A}$.

Thus, the set of all concentration profiles defined by (18)-(19) as a function of all possible values of the parameters ( $\boldsymbol{\mu}, \mathbf{R}, \mathbf{L})$ is identical to the set defined by (15)-(16) as function of $(\boldsymbol{\mu}, \mathbf{M}, \mathbf{V}, \mathbf{L})$. This is a crucial insight, since it allows us to search the entire parameter space using (18)-(19), where the concentration is known explicitly in terms of $(\boldsymbol{\mu}, \mathbf{R}, \mathbf{L})$.

To pose the new optimization problem, it is convenient to define $\bar{v}_{i}^{(j)}=\mu^{(j)} v_{i}^{(j)}$, for $j=$ $1, \ldots, N_{C}$, and $i=1, \ldots, N_{s}$. Furthermore, the glycan distribution the surface is given by $\mu^{N_{C}} v_{i}^{N_{C}}=\bar{v}_{i}$. Thus, it follows that Optimization B:

$$
\bar{D}\left(\sigma, N_{E}, N_{C}, \mathbf{c}^{*}\right):=\min _{\mu, \mathbf{R}, \mathbf{L} \geq \mathbf{0}} F\left(\mathbf{c}^{*} \| \overline{\mathbf{v}}\right)
$$

is equivalent to (6). Since $\mathbf{v}$ is explicitly known as a function of (R, $\mathbf{L})$, optimization B (21) is a more tractable optimization problem than (6). Note that in this setting, the function $D\left(\sigma, N_{E}, N_{C}, \mathbf{c}^{*}\right)$ in (21) is independent of the rates $\mu$. See Fig. 1 for a flow chart of the two optimization schemes.

While 21 is easy to implement, we note that the parameters (e.g., reaction rates, specificity) are not constrained to take only physically relevant values; a legitimate concern is that the absence of such physico-chemical constraints might drive this optimization to physically unrealistic solutions.

There are two possible ways to impose these parameter constraints. One is to impose constraints on the "microscopic" chemical parameters, such as the rate of individual reactions $R(j, k, \alpha)$ and the inter-cisternal transport rate $\mu^{(j)}$. These take into consideration constraints arising from molecular enzymatic processes. The other is to impose constraints on "global" physical parameters, such as the total transport time across the Golgi cisternae and the average enzymatic reaction time. Here, we impose constraints on the microscopic reaction and transport parameters to define constrained Optimization $B$ :

$$
\begin{array}{rl}
\bar{D}\left(\sigma, N_{C}, N_{E}, \mathbf{c}^{*}\right):=\min _{\mu, \mathbf{R}, \mathbf{L}} & F\left(\mathbf{c}^{*} \| \overline{\mathbf{v}}\right) \\
\text { S.t. } & R_{\min } \leq R(j, k, \alpha) \leq R_{\max }, \\
& \mu_{\min } \leq \mu^{(j)} \leq \mu_{\max } \\
& 1 \leq \ell_{\alpha}^{(j)} \leq N_{S} .
\end{array}
$$




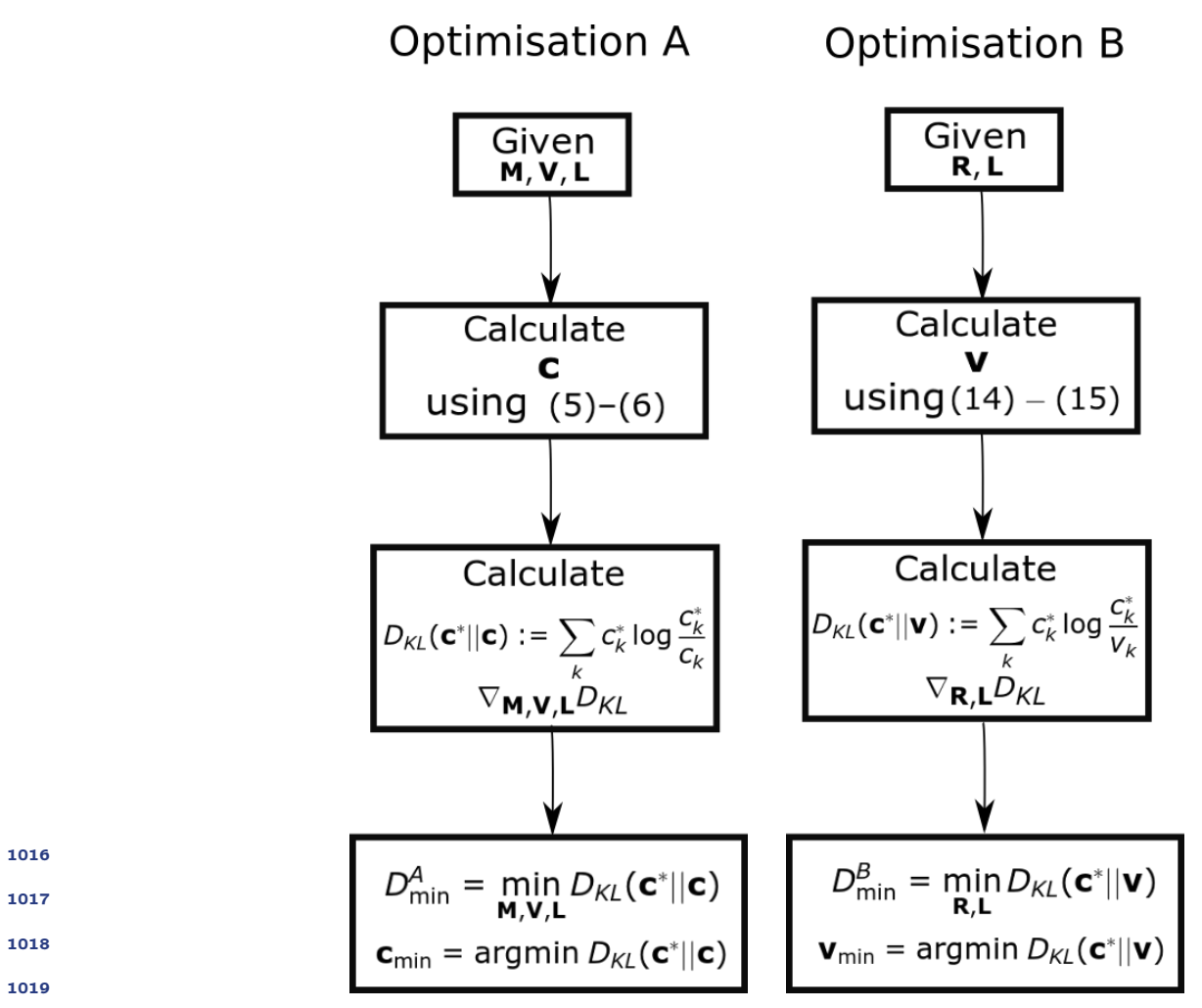

1020

The upper and lower bounds on the rates $\mathbf{R}$ and $\mu$ are estimated in Appendix $2: \mu_{\max }=1 / \mathrm{min}$ (resp. $\mu_{\min }=.01 / \mathrm{min}$ ) and $R_{\max }=20 / \mathrm{min}$ (resp. $R_{\min }=.018 / \mathrm{min}$ ). 


\section{Appendix 5}

\section{Numerical scheme for performing the non-convex optimization}

We solve Optimization C using the numerical scheme detailed below. The optimization problem consists of minimising a non-convex objective with linear box constraints. We use the MATLAB FMINCON function to solve this optimization. We use Sequential Quadratic Programming (SQP), a gradient based iterative optimization scheme for solving optimizations with non-linear differentiable objective and constraints. Since our problem is non-convex and SQP only gives local minima, we initialise the algorithm with many random initial points. We use SOBOLSET function of MATLAB to generate space filling pseudo random numbers. We have taken 1000 initialisations for each $N_{E}, N_{C}$ and $\sigma$ value. We have taken 50 equally spaced points between 0 and 1 to explore the $\sigma$-space for Fig. 3 of the main text. Some minor fluctuations in $D$ due to non-convexity of the objective function in the final results were smoothed out by taking the convex hull of the $D$ vs. $\sigma$ graph. The results for $\sigma_{\min }\left(N_{E}, N_{C}\right)$ and $D\left(\sigma_{\min }, N_{E}, N_{C}\right)$ (Fig. 4 of the main text) were obtained by adding $\sigma$ to the optimization vector and then performing the optimization.

A similar numerical scheme was used to optimize diversity. 
Analytical solution when $N_{s} \gg 1$

It is possible to obtain analytical expressions for the steady state glycan distribution in the limit $N_{s} \gg 1$ when the glycan index $k$ can be approximated by a continuous variable. In this case, (15)-(16) can be cast as differential equations,

$$
\begin{aligned}
& \frac{d c_{k}^{(1)}}{d k} \approx c_{k}^{(1)}-c_{k-1}^{(1)} \\
& =\left(\frac{\sum_{\alpha=1}^{N_{E}} R(1, k-1, \alpha) \exp \left(-\sigma\left|k-1-l_{\alpha}^{(1)}\right|\right)}{\mu^{(1)}+\sum_{\alpha=1}^{N_{E}} R(1, k, \alpha) \exp \left(-\sigma\left|k-l_{\alpha}^{(1)}\right|\right)}-1\right) c_{k-1}^{(1)} \\
& \approx-\left(\frac{\mu^{(1)}+\frac{d}{d k} \sum_{\alpha=1}^{N_{E}} R(1, k, \alpha) \exp \left(-\sigma\left|k-l_{\alpha}^{(1)}\right|\right)}{\mu^{(1)}+\sum_{\alpha=1}^{N_{E}} R(1, k, \alpha) \exp \left(-\sigma\left|k-l_{\alpha}^{(1)}\right|\right)}\right) c_{k}^{(1)},
\end{aligned}
$$

and

$$
\begin{aligned}
\frac{d c_{k}^{(j)}}{d k} & \approx c_{k}^{(j)}-c_{k-1}^{(j)} \\
= & \frac{\mu^{(j-1)}}{\mu^{(j)}+\sum_{\alpha=1}^{N_{E}} R(j, k, \alpha) \exp \left(-\sigma\left|k-l_{\alpha}^{(j)}\right|\right)} c_{k}^{(j-1)} \\
& -\left(\frac{\mu^{(j)}+\frac{d}{d k} \sum_{\alpha=1}^{N_{E}} R(j, k, \alpha) \exp \left(-\sigma\left|k-l_{\alpha}^{(j)}\right|\right)}{\mu^{(j)}+\sum_{\alpha=1}^{N_{E}} R(j, k, \alpha) \exp \left(-\sigma\left|k-l_{\alpha}^{(j)}\right|\right)}\right) c_{k}^{(j)}
\end{aligned}
$$

for $j=2, \ldots, N_{C} \cdot \ln (22)$ and (23),

$$
\begin{aligned}
& \frac{d}{d k} \sum_{\alpha=1}^{N_{E}} R(j, k, \alpha) \exp \left(-\sigma\left|k-l_{\alpha}^{(j)}\right|\right) \\
& \quad=\sum_{\alpha=1}^{N_{E}} R(j, k, \alpha) \sigma \exp \left(-\sigma\left|k-l_{\alpha}^{(j)}\right|\right)\left(1-2 \llbracket\left(k \geq l_{\alpha}\right)\right)+R^{\prime}(j, k, \alpha) \exp \left(-\sigma\left|k-l_{\alpha}^{(j)}\right|\right)
\end{aligned}
$$

where the indicator function $\mathbb{\square}(\cdot)$ is equal to 1 if the argument is true, and zero otherwise and $R^{\prime}(j, k, \alpha)$ is the derivative of $R(j, k, \alpha)$ with respect to $k$.

Define a vector function $C(k) \in \mathbb{R}_{c}^{N}$ of the continuous variable $k$ by $C(k)=\left[c_{k}^{(1)}, c_{k}^{(2)}, \ldots c_{k}^{\left(N_{C}\right)}\right]$. Then (22) and (23) can be written as:

$$
\frac{d C(k)}{d k}=M(k) C(k)
$$

where the matrix $M(k)$ is given by

$$
M(k)=\left[\begin{array}{ccccc}
A^{(1)}(k) & 0 & 0 & 0 & \ldots 0 \\
B^{(2)}(k) & A^{(2)}(k) & 0 & 0 & \ldots 0 \\
0 & B^{(3)}(k) & A^{(3)}(k) & 0 & \ldots 0 \\
\vdots & \vdots & \vdots & \vdots & \vdots \\
0 & \ldots & 0 & B^{\left(N_{C}\right)}(k) & A^{\left(N_{C}\right)}(k)
\end{array}\right]
$$


with

$$
\begin{aligned}
& A^{(j)}(k)=-\frac{\mu^{(j)}+\frac{d}{d k} \sum_{\alpha=1}^{N_{E}} R(j, k, \alpha) \exp \left(-\sigma\left|k-l_{\alpha}^{(j)}\right|\right)}{\mu^{(j)}+\sum_{\alpha=1}^{N_{E}} R(j, k, \alpha) \exp \left(-\sigma\left|k-l_{\alpha}^{(j)}\right|\right)} \\
& B^{(j)}(k)=\frac{\mu^{(j-1)}}{\mu^{(j)}+\sum_{\alpha=1}^{N_{E}} R(j, k, \alpha) \exp \left(-\sigma\left|k-l_{\alpha}^{(j)}\right|\right)}
\end{aligned}
$$

The functions $A^{(j)}(k)$ and $B^{(j)}(k)$ involve absolute value and indicator functions; therefore, the differential equation has to be solved in a piecewise manner assuming continuity of solution $C(k)$.

The general solution of (25)

$$
C(k)=C_{0} \exp (\Omega(k))
$$

is written in terms of the Magnus Function $\Omega(k)=\sum_{n=1}^{\infty} \Omega(n, k)$, obtained from the BakerCampbell-Hausdorff formula Blanes et al. (2009),

$$
\begin{aligned}
& \Omega(1, k)=\int_{0}^{k} M\left(k_{1}\right) d k_{1} \\
& \Omega(2, k)=\frac{1}{2} \int_{0}^{k} d k_{1} \int_{0}^{k_{1}} d k_{2}\left[M\left(k_{1}\right), M\left(k_{2}\right)\right] \\
& \Omega(3, k)=\frac{1}{6} \int_{0}^{k} d k_{1} \int_{0}^{k_{1}} d k_{2} \int_{0}^{k_{2}} d k_{3}\left[M\left(k_{1}\right),\left[M\left(k_{2}\right), M\left(k_{3}\right)\right]\right]+\left[M\left(k_{3}\right),\left[M\left(k_{2}\right), M\left(k_{1}\right)\right]\right]
\end{aligned}
$$

where $\left[M\left(k_{1}\right), M\left(k_{2}\right)\right]:=M\left(k_{1}\right) M\left(k_{2}\right)-M\left(k_{2}\right) M\left(k_{1}\right)$ is the commutator, and the higher order terms in ... contain higher order nested commutators.

Here, we establish conditions under which the series $\sum_{n=1}^{\infty} \Omega(n, k)$ that defines solution $C(k)$ to the differential equation (25) converges. We also solve (25) for some special cases.

The commutator

$$
\left[M\left(k_{1}\right), M\left(k_{2}\right)\right]=\left[\begin{array}{cccccc}
0 & 0 & 0 & 0 & \ldots & 0 \\
a_{21} & 0 & 0 & 0 & \ldots & 0 \\
a_{31} & a_{32} & 0 & 0 & \ldots & 0 \\
0 & a_{42} & a_{43} & 0 & \ldots & 0 \\
\vdots & \vdots & \vdots & \vdots & & \vdots \\
0 & \ldots & & a_{n, n-2} & a_{n, n-1} & 0
\end{array}\right]
$$

where

$$
\begin{aligned}
& a_{i, i-1}=A^{(i-1)}\left(k_{2}\right) B^{(i)}\left(k_{1}\right)+A^{(i)}\left(k_{1}\right) B^{(i)}\left(k_{2}\right)-A^{(i-1)}\left(k_{1}\right) B^{(i)}\left(k_{2}\right)+A^{(i)}\left(k_{2}\right) B^{(i)}\left(k_{1}\right) \\
& a_{i, i-2}=B^{(i-1)}\left(k_{2}\right) B^{(i)}\left(k_{1}\right)-B^{(i-1)}\left(k_{1}\right) B^{(i)}\left(k_{2}\right)
\end{aligned}
$$

The general form of $\Omega(n, k)$ is given by Blanes et al. (2009)

$$
\Omega(n, k)=\frac{z_{n}}{n !} \int_{0}^{k} d k_{1} \int_{0}^{k_{1}} d k_{2} \ldots \int_{0}^{k_{n-2}} d k_{n-1} \int_{0}^{k_{n-1}} d k_{n} \sum_{l} W_{l} M\left(k_{p_{1}^{\prime}}\right) M\left(k_{p_{2}^{\prime}}\right) \ldots M\left(k_{p_{n}^{\prime}}\right)
$$

where $\left(p_{1}^{(l)}, p_{2}^{(l)} \ldots p_{n}^{(l)}\right)$ is a permutation of $(1,2,3, \ldots n), W_{l} \in\{-1,1\}$, and $z_{n} \in 1, \ldots n$. 
Let $\bar{A}=\max _{k, l, m}\left|M_{l, m}(k)\right|$. Define

$$
\bar{M}=\left[\begin{array}{ccccc}
\bar{A} & 0 & 0 & 0 \ldots & 0 \\
\bar{A} & \bar{A} & 0 & 0 \ldots & 0 \\
0 & \bar{A} & \bar{A} & 0 \ldots & 0 \\
\vdots & \vdots & \vdots & & \vdots \\
0 & \ldots & 0 & \bar{A} & \bar{A}
\end{array}\right]
$$

We can bound all the matrix elements of $\Omega(n, k)$ in the following way

$$
\begin{aligned}
\Omega_{l m}(n, k) & \leq z_{n} \bar{M}_{l, m}^{n} \int_{0}^{k} d k_{1} \int_{0}^{k_{1}} d k_{2} \ldots \int_{0}^{k_{n-1}} d k_{n} \\
& =\left.z_{n} \bar{M}^{n}\right|_{l m} \frac{k^{n}}{n !}
\end{aligned}
$$

The matrix

$$
\bar{M}^{n}=\left[\begin{array}{cccccc}
a_{11} & 0 & 0 & 0 & \ldots & 0 \\
a_{21} & a_{22} & 0 & 0 & \ldots & 0 \\
a_{31} & a_{32} & a_{33} & 0 & \ldots & 0 \\
a_{41} & a_{42} & a_{43} & a_{44} & \ldots & 0 \\
\vdots & \vdots & \vdots & \vdots & & \vdots \\
a_{n 1} & \ldots & & a_{n, n-2} & a_{n, n-1} & a_{n n}
\end{array}\right]
$$

where $a_{l m}=S_{l m}(n) \bar{A}^{n}$ for appropriately defined polynomials $S_{l, m}(n)$. Thus, it follows that $\Omega_{l m} \leq$ $z_{n} S_{l m}(n)\left(A^{*}\right)^{n} \frac{k^{n}}{n !}$ and $\Omega_{l, m}(k) \leq \sum_{n=1}^{\infty} z_{n} S_{l, m}(n)\left(A^{*}\right)^{n} \frac{k^{n}}{n !}$. Consequently, the series will converge if $\bar{A} k<1$, i.e. $k \leq \frac{1}{\bar{A}}$. Assuming $\mu^{(j)}=\mu \forall j$, we can bound $\bar{A}$ as

$$
\bar{A} \leq \max _{j, k}\left(\frac{\mu+\sigma \sum_{\alpha=1}^{N_{E}} R(j, k, \alpha) \exp \left(-\sigma\left|k-l_{\alpha}^{(j)}\right|\right)}{\mu+\sum_{\alpha=1}^{N_{E}} R(j, k, \alpha) \exp \left(-\sigma\left|k-l_{\alpha}^{(j)}\right|\right)}+\frac{\sum_{\alpha=1}^{N_{E}} R^{\prime}(j, k, \alpha) \exp \left(-\sigma\left|k-l_{\alpha}^{(j)}\right|\right)}{\mu+\sum_{\alpha=1}^{N_{E}} R(j, k, \alpha) \exp \left(-\sigma\left|k-l_{\alpha}^{(j)}\right|\right)}\right)
$$

Since the parameters $\mu, \sigma, R(j, k, \alpha), l_{\alpha}^{(j)}$ and $N_{E}$ are finite and positive, and $R^{\prime}(j, k, \alpha)$ is finite, $\bar{A}$ has a finite upper bound, implying $k$ is always greater than zero, and the series has finite radius of convergence.

While in principle we can obtain the glycan profile for any $N_{E}$ and $N_{C}$ with arbitrary accuracy, assuming $R(j, k, \alpha)=R_{\alpha}^{(j)}$, we provide explicit formulae for a few representative cases : (i) $\left(N_{E}=1, N_{C}=1\right)$ and (ii) $\left(N_{E}=1, N_{C}=2\right)$.

(i) $N_{E}=1, N_{C}=1$ : The solution of the differential equation is given by

$$
c(k)= \begin{cases}c_{0} e^{-k}\left(\frac{\mu+R \exp (-\sigma(l-k))}{\mu+R \exp (-\sigma l)}\right)^{(1 / \sigma)-1} & k \leq l \\ c(l) e^{-(k-l)}\left(\frac{\mu+R}{\mu+R \exp (-\sigma(k-l))}\right)^{(1 / \sigma)+1} & k>l\end{cases}
$$

A representative concentration profile is plotted in Appendix 6 Fig. 1. The concentration profile consists of two distinct components: an initial exponential decay, and then an exponential rise and fall concentrated around $l$. The relative weight of these two components is controlled by the sensitivity $\sigma$ and the rate $R$. Such explicit formulae can be obtained for any $N_{E}>1$, as long as $N_{C}=1$.

(ii) $N_{E}=1, N_{C}=2$ : The concentration profile $c^{(2)}$ in cisterna 2 can be obtained from the following calculation. Let $l^{(j)}$ denote the "length" of the enzyme in cisterna $j=1,2$. For 
$k \leq \min \left\{l^{(1)}, l^{(2)}\right\}$

$$
\begin{aligned}
c^{(2)}(k)= & c_{0} \mu^{(1)} e^{-k}\left(\frac{\mu^{(2)}+R^{(2)} \exp \left(-\sigma\left(l^{(2)}-k\right)\right)}{\mu^{(1)}+R^{(1)} e^{-\sigma l^{(1)}}}\right)^{(1 / \sigma)-1} \int_{0}^{k} \frac{\left(\mu^{(1)}+R^{(1)} \exp \left(-\sigma\left(l^{(1)}-k\right)\right)\right)^{(1 / \sigma)-1}}{\left(\mu^{(2)}+R^{(2)} \exp \left(-\sigma\left(l^{(2)}-k\right)\right)\right)^{1 / \sigma}} d k \\
& +c^{(2)}(0) e^{-k}\left(\frac{\mu^{(2)}+R^{(2)} e^{-\sigma\left(l^{(2)}-k\right)}}{\mu^{(2)}+R^{(2)} e^{-\sigma l^{(2)}}}\right)^{(1 / \sigma)-1}
\end{aligned}
$$

Next, consider the case where $l^{(1)} \leq l^{(2)}$. Then, for $l^{(1)}<k \leq l^{(2)}$

$$
\begin{aligned}
c^{(2)}(k)= & c^{(1)}\left(l^{(1)}\right) \mu^{(1)} e^{-\left(k-l^{(1)}\right)}\left(\mu^{(1)}+R^{(1)}\right)^{(1 / \sigma)+1}\left(\mu^{(2)}+R^{(2)} \exp \left(-\sigma\left(l^{(2)}-k\right)\right)\right)^{(1 / \sigma)-1} \\
& \int_{l^{(1)}}^{k} \frac{\left(\mu^{(2)}+R^{(2)} \exp \left(-\sigma\left(l^{(2)}-k\right)\right)\right)^{-1 / \sigma}}{\left(\mu^{(1)}+R^{(1)} \exp \left(-\sigma\left(k-l^{(1)}\right)\right)\right)^{(1 / \sigma)+1}} d k \\
+ & c^{(2)}\left(l^{(1)}\right) e^{-\left(k-l^{(1)}\right)}\left(\frac{\mu^{(2)}+R^{(2)} e^{-\sigma\left(l^{(2)}-k\right)}}{\mu^{(2)}+R^{(2)} e^{-\sigma\left(l^{(2)}-l^{(1)}\right)}}\right)^{(1 / \sigma)-1}
\end{aligned}
$$

and for $l^{(1)} \leq l^{(2)}<k$,

$$
\begin{aligned}
c^{(2)}(k)= & c^{(1)}\left(l^{(1)}\right) \mu^{(1)} e^{-\left(k-l^{(1)}\right)}\left(\frac{\mu^{(1)}+R^{(1)}}{\mu^{(2)}+R^{(2)} \exp \left(-\sigma\left(k-l^{(2)}\right)\right)}\right)^{(1 / \sigma)+1} \\
& \int_{l^{(2)}}^{k} \frac{\left(\mu^{(2)}+R^{(2)} \exp \left(-\sigma\left(k-l^{(2)}\right)\right)\right)^{1 / \sigma}}{\left(\mu^{(1)}+R^{(1)} \exp \left(-\sigma\left(k-l^{(1)}\right)\right)\right)^{(1 / \sigma)+1}} d k \\
+ & c^{(2)}\left(l^{(2)}\right) e^{-\left(k-l^{(2)}\right)}\left(\frac{\mu^{(2)}+R^{(2)}}{\mu^{(2)}+R^{(2)} e^{-\sigma\left(k-l^{(2)}\right)}}\right)^{(1 / \sigma)+1}
\end{aligned}
$$

Next, the case where $l^{(1)} \geq l^{(2)}$. For $l^{(2)}<k \leq l^{(1)}$,

$$
\begin{aligned}
c^{(2)}(k) & =c_{0} \mu^{(1)} e^{-k} \frac{\left(\mu^{(1)}+R^{(1)} e^{-\sigma l^{(1)}}\right)^{1-(1 / \sigma)}}{\left(\mu^{(2)}+R^{(2)} \exp \left(-\sigma\left(k-l^{(2)}\right)\right)\right)^{(1 / \sigma)+1}} \int_{l^{(2)}}^{k} \frac{\left(\mu^{(1)}+R^{(1)} \exp \left(-\sigma\left(l^{(1)}-k\right)\right)\right)^{(1 / \sigma)-1}}{\left(\mu^{(2)}+R^{(2)} \exp \left(-\sigma\left(k-l^{(2)}\right)\right)\right)^{-1 / \sigma}} d k \\
& +c^{(2)}\left(l^{(2)}\right) e^{l^{(2)}-k}\left(\frac{\mu^{(2)}+R^{(2)}}{\mu^{(2)}+R^{(2)} e^{-\sigma\left(k-l^{(2)}\right)}}\right)^{(1 / \sigma)+1}
\end{aligned}
$$

For $^{(2)} \leq l^{(1)}<k$,

$$
\begin{aligned}
c^{(2)}(k)= & c^{(1)}\left(l^{(1)}\right) \mu^{(1)} e^{-\left(k-l^{(1)}\right)}\left(\frac{\mu^{(1)}+R^{(1)}}{\mu^{(2)}+R^{(2)} \exp \left(-\sigma\left(k-l^{(2)}\right)\right)}\right)^{(1 / \sigma)+1} \\
& \int_{l^{(2)}}^{k} \frac{\left(\mu^{(2)}+R^{(2)} \exp \left(-\sigma\left(k-l^{(2)}\right)\right)\right)^{1 / \sigma}}{\left(\mu^{(1)}+R^{(1)} \exp \left(-\sigma\left(k-l^{(1)}\right)\right)\right)^{(1 / \sigma)+1}} d k \\
+ & c^{(2)}\left(l^{(1)}\right) e^{-\left(k-l^{(1)}\right)}\left(\frac{\mu^{(2)}+R^{(2)} e^{-\sigma\left(l^{(1)}-l^{(2)}\right)}}{\mu^{(2)}+R^{(2)} e^{-\sigma\left(k-l^{(2)}\right)}}\right)^{(1 / \sigma)+1}
\end{aligned}
$$

The integrals in (32) to (36) can evaluated numerically. The result of the numerical computation is shown in Fig. 1.

Fig. 2 (a)-(d) plots the glycan profile $c_{k} v s . k$ as one varies the enzyme specificity $\sigma$, the reaction rates $R$ and transport rates $\mu$, for two different values of $N_{E}$ and $N_{C}$. The results in the plots lead us to the following general observations:

(i) Very low specificity enzymes cannot generate complex glycan distributions. Keeping everything else fixed, intermediate or high specificity enzymes can generate glycan distributions of higher complexity by increasing $N_{E}$ or $N_{C}$ (Figs. 2 (a),(c)). 
(a)

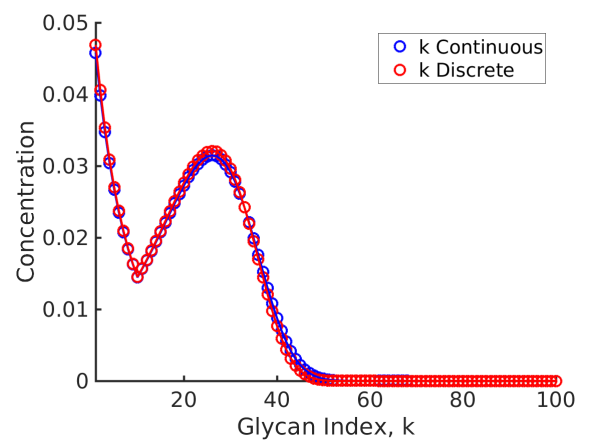

(b)

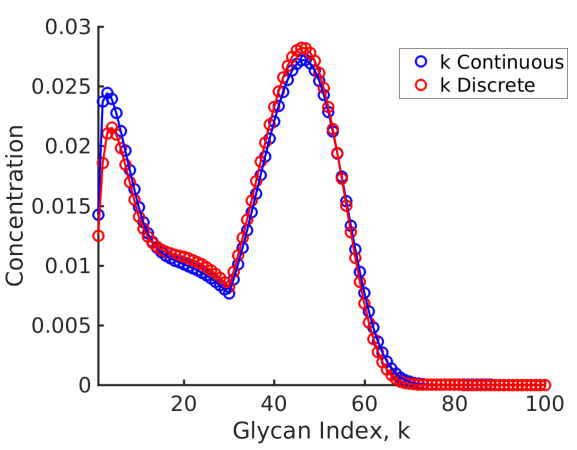

Figure 1. Glycan concentration profile calculated from the model using (a) formula (31) for $N_{E}=N_{C}=1$ and (b) formulae (32)-(36) for $N_{E}=1, N_{C}=2$.

(ii) Decreasing the specificity $\sigma$ or increasing the rates $R$ increases the proportion of higher index glycans. Keeping everything else fixed, changes in the rate $R$ have a stronger impact on the relative weights of the higher index glycans to lower index glycans. The relative weight of the higher index glycans increases with increasing $N_{E}$ and $N_{C}$ (Figs. 2 (b)-(d)).

(iii) Keeping everything else fixed, decreasing enzyme specificity increases the spread of the distribution around the peaks (Appendix 6 Figs. 2 (a),(c)). 
(a)

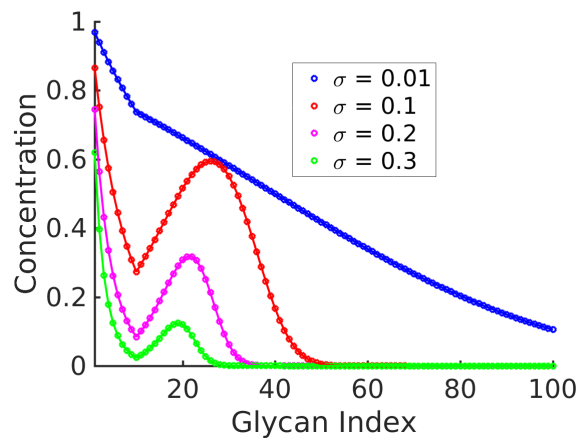

(c)

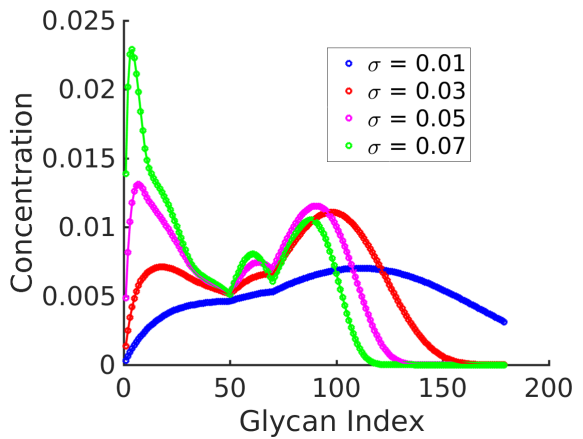

(b)

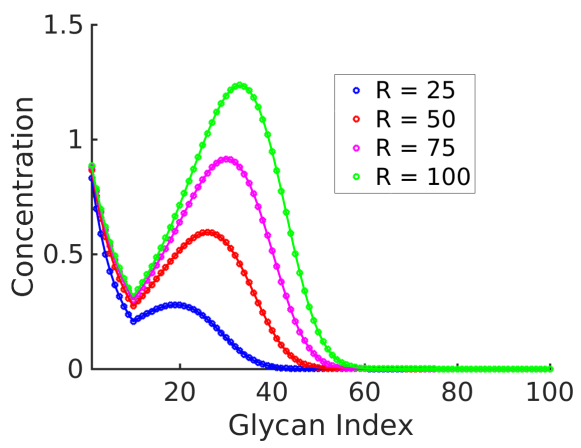

(d)

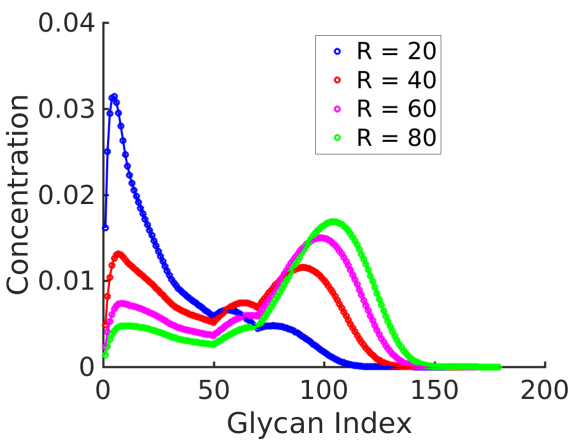

Figure 2. Glycan profile $\left\{c_{k}: k=1, \ldots, N_{s}\right\}$ as a function of specificity $\sigma$ (Fig. (a), (c)), and reaction rates $R$ (Fig. (b), (d)).

Fig. (a): $N_{E}=N_{C}=1,(R=50, \mu=1, l=10) . c_{k}$ decreases exponentially with $k$ for very low and very high $\sigma$; however, the decay rate is lower at low $\sigma$. For intermediate values of $\sigma$, the distribution has exactly two peaks, one of which is at $k=0$, and eventually decays exponentially. The width of the distribution is a decreasing function of $\sigma$.

Fig. (b): $N_{E}=N_{C}=1,(\sigma=0.1, \mu=1, l=10)$. At low $R, c_{k}$ is concentrated at low $k$. The proportion of higher index glycans in an increasing function of $R$.

Fig. (c): $N_{E}=N_{C}=2,\left(R=40, \mu=1,\left[l_{1}^{(1)}, l_{2}^{(1)}, l_{1}^{(2)}, l_{2}^{(2)}\right]=[10,30,50,70]\right)$. As $\sigma$ increases, the distribution becomes more complex - from a single peaked distribution at low $\sigma$ to a maximum of four-peaked distribution at high $\sigma$. The peaks gets sharper, and more well defined as $\sigma$ increases.

Fig. (d): $N_{E}=N_{C}=2,\left(R=40, \mu=1,\left[l_{1}^{(1)}, l_{2}^{(1)}, l_{1}^{(2)}, l_{2}^{(2)}\right]=[10,30,50,70]\right)$. As in the plots in Fig. (b), increasing $R$ shifts the peaks towards higher index glycans and the proportion of higher index glycan increases. 
(a) $l_{b} / N_{S}=0.01$

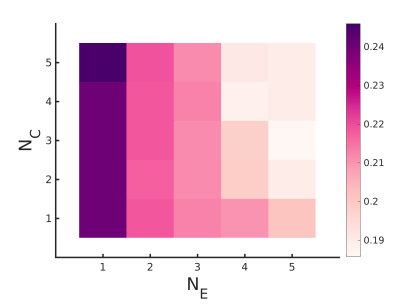

(b) $l_{b} / N_{S}=0.1$

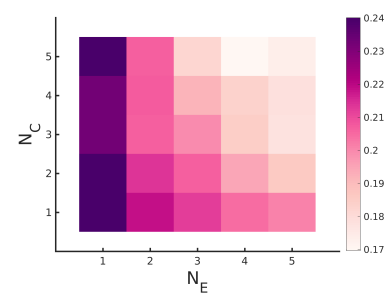

(c) $l_{b} / N_{S}=0.5$

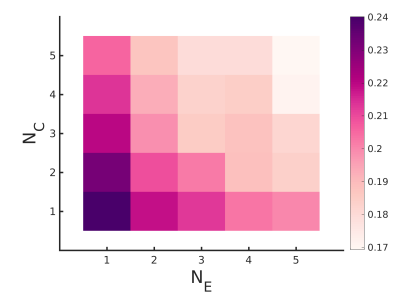

Figure 1. Optimum fidelity $\bar{D}_{K L}$ as a function of $\left(N_{E}, N_{C}\right)$ for different values of $\ell_{b} / N_{s}$, where $\ell_{b}$ bounds the deformation in the ideal length $\ell_{\alpha}^{(0)}$ of an enzyme $\alpha=1, \ldots, N_{E}$. Small values of $\ell_{b}$ restricts all enzymes from working in all cisternae and all substrates, where large value of $\ell_{b}$ removes this constraint.

\section{Appendix 7}

\section{Extended distortion model shows lack of apparent symmetry}

The results in Fig. 4 seem to imply that there is an approximate $N_{E}-N_{C}$ symmetery in the model, i.e. increasing either $N_{E}$ or $N_{C}$ affects the fidelity, optimal enzyme specificity and the sensitivity in approximately the same way. This is a consequence of the distortion model we are using for calculating the binding probabilities of substrates with enzymes, which allows every enzyme $\alpha$ to in principle catalyse any reaction in any cisterna. This allowed for the ideal enzyme length $l_{\alpha}^{(j)}$ in Equation 3 to vary across the cisternae in an unconstrained manner, leading to simplification in the calculation. We now find that by changing this aspect of the model, the apparent symmetry between $N_{E}-N_{C}$ is lifted. A more reasonable model for the ideal enzyme length is given by

$$
\ell_{\alpha}^{(j)}=\ell_{\alpha}^{(0)}+\delta \ell_{\alpha}^{(j)}, \quad \delta \ell_{\alpha}^{(j)} \in\left[-\ell_{b}^{(j)}, \ell_{b}^{(j)}\right],
$$

i.e., the nominal length $\ell_{\alpha}^{(0)}$ can be distorted in a cisterna by a correction $\delta \ell_{\alpha}^{(j)}$ but within a specified bound $\ell_{b}^{(j)}$ that is not subject to optimization. One can render some enzymes inactive in certain cisternae by choosing appropriate values of $\ell_{\alpha}^{(0)}$ and $\delta \ell_{\alpha}^{(j)}$. For small values for the bound $\ell_{b}$, e.g $l_{b} / N_{s} \leq 0.2$ (here $N_{s}-1$ is the number of enzymatic reactions), the decrease in $\bar{D}$ on increasing $N_{C}$ is small compared to increasing $N_{E}$ (see Fig. 1). On the other hand for large $\ell_{b}$, e.g. $l_{b} / N_{S} \geq 0.3$, there is an approximate symmetry between $N_{E}$ and $N_{C}$ (see Fig. 1). Here we have taken the bounds to be compartment independent, i.e. $\ell_{b}^{(j)}=\ell_{b}$. 


\section{Appendix 8}

\section{Redundancies and non-convexity of the optimization}

\section{Validation of the numerical optimization scheme.}

In order to test whether our numerical optimization procedure is able to converge to the global minimum we run the following test. We generate 100 random values of $(\boldsymbol{\mu}, \boldsymbol{R}, \boldsymbol{L}, \sigma)$ within their respective ranges for a problem instance with $\left(N_{E}=2, N_{C}=2\right)$. The sampled value for $(\boldsymbol{\mu}, \boldsymbol{R}, \boldsymbol{L}, \sigma)$ is used to generate concentration profiles that are then used as the target distribution for the optimization. Since the target distribution is achievable, the optimal value of the constrained Optimization $B$ for these sampled targets is $\bar{D}=0$. We solve the constrained Optimization $B$ using our numerical scheme. The average optimal value $\bar{D}$ across all sampled values was $9.1835 \mathrm{e}-07,30$ out of 100 values were exactly zero, and the highest $\bar{D}$ was $1.1761 \mathrm{e}-05$. Therefore, the optimization scheme was able to recover the concentration profiles almost exactly. Next, we ask whether the optimization problem recovers the value of $(\boldsymbol{\mu}, \boldsymbol{R}, \boldsymbol{L}, \sigma)$ that was used to create the particular target distribution. We were able to recover $\sigma$ exactly, except in cases where the concentration profile was almost a delta function at the first glycan (see Fig. 1). This is because $\sigma$ decides the typical width of the empirical distribution, and hence the optimal $\sigma$ is determined by the typical width of the target distribution, except in the pathological case of a concentration profile that is almost a delta function at the first glycan - such a concentration profile can be made produced for any value $\sigma$ by simply making transport $\mu$ very fast as compared to the reaction rates.

We note that the optimization in $(\boldsymbol{\mu}, \boldsymbol{R}, \boldsymbol{L})$ is not convex, and leads to many equally good minimas corresponding to different values of $(\boldsymbol{\mu}, \boldsymbol{R}, \boldsymbol{L})$. The resulting redundancies in the model and their importance are discussed next.

\section{Degeneracy in the model}

Recall that in equation 7 , we defined

$$
R_{e f f}(j, k)=\frac{\sum_{\alpha} R_{\alpha}^{(j)} \exp \left(-\sigma\left|k-l_{\alpha}^{(j)}\right|\right)}{\mu^{(j)}}
$$

In terms of these renormalised rates, the steady glycan concentration can be written as

$$
\bar{c}_{k}^{(j)}=\frac{R_{e f f}(j, k) \bar{c}_{k-1}^{(j)}+\bar{c}_{k}^{j-1}}{1+R_{e f f}(j, k)},
$$

i.e. the concentration is only a function of $R_{e f f}(k, j)$. Thus, any combination of $(\boldsymbol{\mu}, \boldsymbol{R}, \boldsymbol{L}, \sigma)$ that maps to the same value of $R_{\text {eff }}$ will result in the same concentration profile, and will be indistinguishable from the perspective of the objective function. Additionally, the mapping from $\mathbf{R}_{e f f}$ to the concentration profile $\bar{c}$ also has degeneracy. We show these redundancies in the schematic below, which shows a systematic reduction in dimension to 1 (scalar) which is the quantity we optimize,

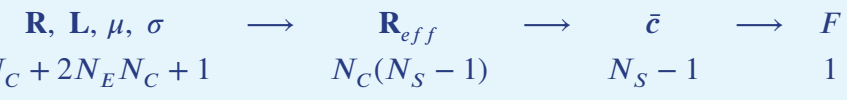


Since $F\left(\boldsymbol{c}_{T} \| \overline{\boldsymbol{c}}\right)=0$ if, and only if, $\boldsymbol{c}_{T}=\overline{\boldsymbol{c}}$, it follows that the last mapping does not have redundancy. Some of the sources of degeneracies in the mapping from $(\boldsymbol{\mu}, \boldsymbol{R}, \boldsymbol{L}, \sigma)$ to $\boldsymbol{R}_{\text {eff }}$ are as follows:

1. For fixed $(\sigma, \boldsymbol{L})$, setting $R_{\alpha}^{(j)} \leftarrow \gamma \boldsymbol{R}_{\alpha}^{(j)}$ and $\mu_{j} \leftarrow \gamma^{-1} \mu_{j}$ leaves $\boldsymbol{R}_{e f f}$ invariant.

2. Permutations in the $\alpha$ index leaves $R_{\text {eff }}$ invariant. Thus, there are at least $\left(N_{E} !\right)^{N_{C}}$ distinct minima that map to the same value of $R_{e f f}$, and therefore, the same concentration $\bar{c}$.

Additionally, there are degeneracies coming from the optimization which depend on the target distribution $c_{T}$. Having discussed the sources of degeneracies of the optimized solution, we now discuss the distribution of the optimized solutions.

\section{Distribution of minima}

To study the behaviour of the optimization algorithm for different initial points, we numerically investigate the distribution of function values at different local minima. Since the dimension of the optimization problem is $N_{C}+2 N_{E} N_{C}+1$, which is large, we divide the optimization space into a grid of $I=n_{p}^{\left(N_{C}+2 N_{E} N_{C}+1\right)}$ points. We did this numerical experiment for $\left(N_{E}, N_{C}\right) \in\{(1,1),(1,2),(2,1),(2,2)\}$. The value of $n_{p}=3$ for $\left(N_{E}, N_{C}\right)=(2,2)$ and $n_{p}=4$ for the rest. The target distribution for all the cases is a single Gaussian with mean 20, standard deviation 5 , with support on $1 \leq k \leq 20$. The results of this numerical experiment are summarized in Fig. 2 and Table 1, from which we deduce the following:

1. A large fraction of the initial starting points converge to a set of degenerate minima with objective function value exactly equal to the global minimum. These minima are a result of the degeneracies of the optimization problem.

2. There are other local minima with objective value very close to (but not equal) to the global minimum.

Most initial points converge to one of these two sets of minima.

3. Finally, there are a small set of local minima with significantly higher objective values. These correspond to minima with $\sigma=0$. The fraction of initial points that converge to such minima reduces as the dimension of the optimization space increases.

\begin{tabular}{l|l|l|l|l}
\hline$N_{E}$ & $N_{C}$ & $\min \bar{D}_{K L}$ & $\max \bar{D}_{K L}$ & $\begin{array}{l}\text { Fraction of initial conditions within } \\
\bar{D}_{K L} \leq 0.0228\end{array}$ \\
\hline \hline 1 & 1 & 0.0228 & 0.44 & 0.56 \\
2 & 1 & 0.0081 & 0.44 & 0.73 \\
1 & 2 & 0.0051 & 0.29 & 0.70 \\
2 & 2 & $1.17 \mathrm{e}-4$ & 0.29 & 0.84 \\
\hline
\end{tabular}

Table 1. Distribution of local minima 

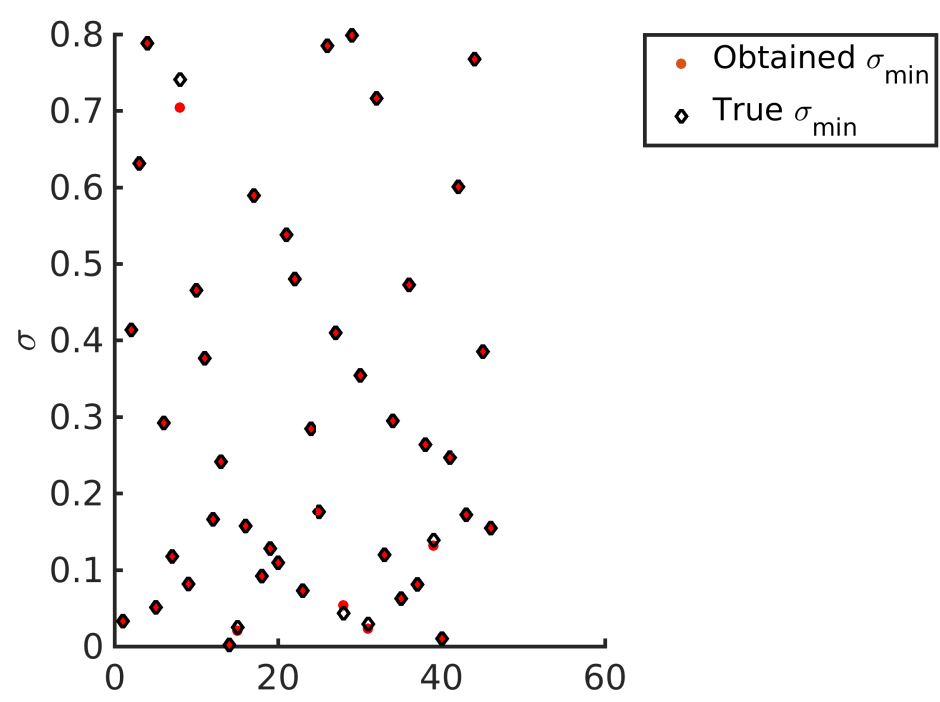

Figure 1. Recovering the $\sigma$ values for different target distribution. Note that barring 4 data points, all other optimized $\sigma$ values (red dots) exactly overlap with the corresponding target $\sigma$ (diamonds).

(a) $N_{E}=1, N_{C}=1$

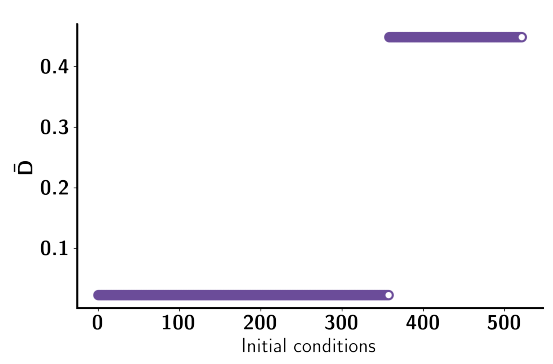

(c) $N_{E}=1, N_{C}=2$

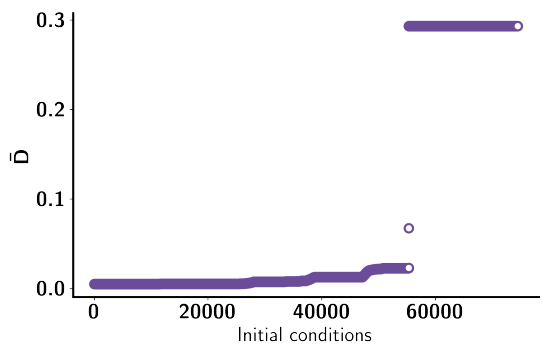

(b) $N_{E}=2, N_{C}=1$

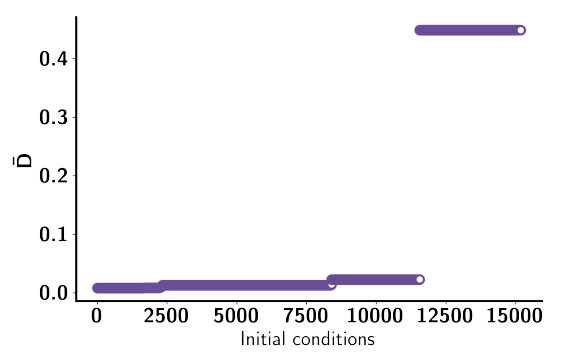

(d) $N_{E}=2, N_{C}=2$

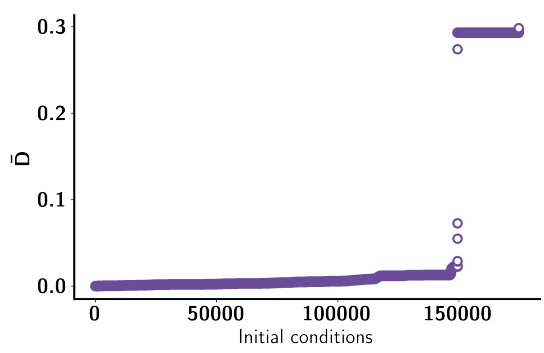

Figure 2. $\bar{D}$ for various initial conditions, sorted in increasing order for clarity. This clearly shows the fraction of initial conditions for which the optimised $\bar{D}$ is small (see Table 1). 


\section{Appendix 9}

\section{Robustness of optimization to small perturbations}

We analyze the sensitivity to small perturbations around the optimal point by calculating the Hessian of the KL divergence,

$$
H(i, j)=\left.\frac{\partial^{2}}{\partial X_{i} \partial X_{j}} F\right|_{X_{\min }}
$$

Here $X=[\boldsymbol{\mu}, \boldsymbol{R}, \boldsymbol{L}, \sigma]$ denotes the entire set of optimization variables (note that the enteries in $X$ are normalized by their respective range and do not carry physical dimensions). We calculated the eigenvalues, denoted by $\lambda_{i}$, and eigenvectors, denoted by $\boldsymbol{V}_{i}$ of the Hessian matrix to identify the stiff and sloppy directions (Gutenkunst et al., 2007; Machta et al., 2013) in the optimization space. The eigenvectors of the Hessian matrix can be grouped in $\boldsymbol{R}, \boldsymbol{L}, \boldsymbol{\mu}$ and $\sigma$ directions by looking for the most dominant component in the eigenvector. We find that most of the eigenvectors have significant entries along the direction of only one of the optimization variables $\boldsymbol{\mu}, \boldsymbol{R}, \boldsymbol{L}, \sigma$, e.g.. in Fig. 6(a), the eigenvectors $21-36$ have significant entries only in the $\boldsymbol{L}$ directions. There is however a small number of eigenvectors that have entries over more than one optimization direction, e.g., the eigenvector with $\sigma$ dominant direction has some $\boldsymbol{\mu}$ component as well (Fig. 6 (a) of the main text).

\section{Stiff and sloppy directions:}

We find that the eigenvalues of the eigenvectors dominated by $\sigma$ and some $\boldsymbol{\mu}, \mathbf{L}$ directions are orders of magnitude higher than for those dominated by the $\boldsymbol{R}$ directions (See Fig. 6 (b) of the main text). This suggests $\bar{D}$ has a valley-like structure around the optimal, with $\boldsymbol{R}$ and some $\boldsymbol{L}$ being the flat or sloppy directions.

The fact that enzyme specificity $\sigma$ and some of the $\mathbf{L}$ directions are stiff should not be surprising, since the typical width and position of peaks in the synthesized distribution is primarily controlled by $\sigma$ and $\mathbf{L}$. We have already shown that $\bar{D}$ is a sharp convex function of $\sigma$ for low values of $\left(N_{E}, N_{C}\right)$ (see Fig. 3 of the main text), which gradually flattens out as we increase $\left(N_{E}, N_{C}\right)$.

The fact that transport rate $\mu$ is a stiff direction is surprising! The stiffness in $\mu$ is due to the fact that the optimal $\mu$ is always at the lower bound, and with even slight increase in $\boldsymbol{\mu}$, the transport becomes too fast for the reactions to be able to produce the intermediate products. For the $(\boldsymbol{R}, \boldsymbol{L})$-dominated eigenvectors, there are bands of sloppy direction and stiff directions. We define the average stiffness in $\mu, \boldsymbol{R}, \boldsymbol{L}$ and $\sigma$ by a weighted average of eigenvalues, where the weight is given by the strength of the corresponding components of the eigenvector.

$$
\langle\lambda\rangle_{\mu}=\ln \left(\sum_{i} w_{i}^{(\mu)} \lambda_{i}\right),\langle\lambda\rangle_{R}=\ln \left(\sum_{i} w_{i}^{(R)} \lambda_{i}\right),\langle\lambda\rangle_{L}=\ln \left(\sum_{i} w_{i}^{(L)} \lambda_{i}\right),\langle\lambda\rangle_{\sigma}=\ln \left(\sum_{i} w_{i}^{(\sigma)} \lambda_{i}\right)
$$

Here $w_{i}^{(\mu)}=\sum_{j \in \mu}\left|V_{i, j}\right| / \sum_{j}\left|V_{i, j}\right|, w_{i}^{(R)}=\sum_{j \in \boldsymbol{R}}\left|V_{i, j}\right| / \sum_{j}\left|V_{i, j}\right|, w_{i}^{(L)}=\sum_{j \in L}\left|V_{i, j}\right| / \sum_{j}\left|V_{i, j}\right|$ and $w_{i}^{(\mu)}=\sum_{j \in \sigma}\left|V_{i, j}\right| / \sum_{j}\left|V_{i, j}\right|$.

Fig 6 (c) of the main text shows $\langle\lambda\rangle_{\mu^{\prime}}\langle\lambda\rangle_{R^{\prime}}\langle\lambda\rangle_{L}$ and $\langle\lambda\rangle_{\sigma}$ as a function of $N_{C}$ for fixed $N_{E}=4$. The average stiffness in $\boldsymbol{R}$ directions, $\langle\lambda\rangle_{R^{\prime}}$ is considerably lower than the average stiffness 

in $\sigma, \boldsymbol{\mu}$ and $\boldsymbol{L}$ directions. $\sigma$ is the stiffest direction but the stiffness decreases on increasing the $N_{C}$. Interestingly, the stiffness along $\boldsymbol{L}$ directions increases on increasing $N_{C}$. eigenvalues divided by the dimension of the optimization problem, in the space of $N_{E}, N_{C}$. We find that the average stiffness is higher for low values of $\left(N_{E}, N_{C}\right)$ as compared to higher values of $\left(N_{E}, N_{C}\right)$, with a few exceptions; and eventually, the average stiffness settles to a fixed low value (Fig. 6 (d) of the main text). 
(a) $c_{t h}=\frac{1}{N_{s}}$

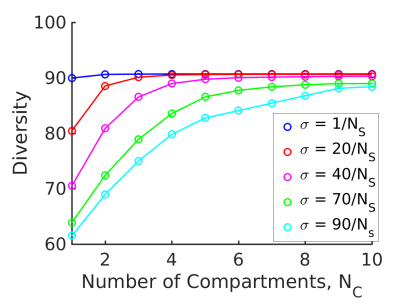

(b) $c_{t h}=\frac{1}{2 N_{s}}$

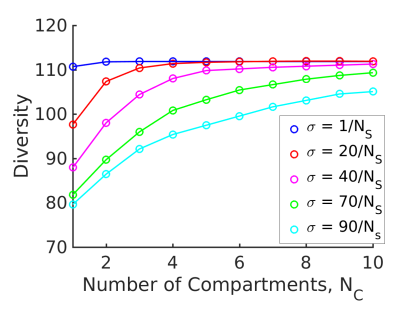

(c) $c_{t h}=\frac{1}{4 N_{s}}$

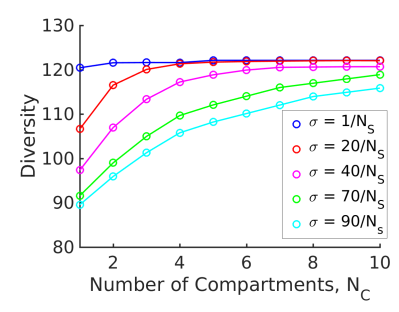

Figure 1. Diversity vs. $N_{C}$ for different values of $\sigma$ keeping $N_{E}=1$ fixed, for three different values of the threshold, $c_{t h}=\frac{1}{N_{s}}, \frac{1}{2 N_{s}}, \frac{1}{4 N_{s}}$. Changing the value of the threshold $c_{t h}$, only changes the saturation value of the diversity curve.

${ }_{1314}$ Appendix 10

1315 Insensitivity of Diversity to threshold $c_{t h}$

Since the threshold $c_{t h}$ used to count the number of glycan species in the definition of $d i$ versity is arbitrary (see, Sect. 8), we here show that the qualitative results we obtain are independent of this choice( Fig. 1). 
1319 Appendix 11

1320

Tables of symbols used in the paper

\begin{tabular}{|l|l|}
\hline symbol & Definition \\
\hline$c_{k}^{(j)}$ & concentration of $k$-th glycan in $j$-th compartment \\
$\mu^{(j)}$ & transport rate from $j$-th to $j+1$ compartment \\
$\sigma$ & specificity of the enzymes \\
$\ell_{\alpha}^{(j)}$ & ideal substrate length for $\alpha$-th enzyme in $j$-th compartment \\
$M(j, k, \alpha)$ & Enzyme parameter related to the Michaelis constant $K_{M}$ \\
$V(j, k, \alpha)$ & Enzyme parameter related to $V_{\text {max }}$ \\
$R(j, \alpha)$ & Reaction parameter for optimization $B$ \\
$D\left(\mathbf{c}^{*} \| \mathbf{c}\right)$ & KL Divergence between $\mathbf{c}^{*}$ and $\mathbf{c}$ \\
$F\left(\mathbf{c}^{*} \| \mathbf{c}\right)$ & Fidelity, KL Divergence normalized by the entropy of the target $\mathbf{c}^{*}$ \\
$\bar{D}\left(\sigma, N_{E}, N_{C}, \mathbf{c}^{*}\right)$ & $\min _{\boldsymbol{\mu}, \boldsymbol{R}, \boldsymbol{L}} F\left(\mathbf{c}^{*} \| \mathbf{c}\right)$ \\
$R_{e f f}(j, k)$ & Effective reaction rate of $k$-th reaction in $j$-th compartment \\
\hline
\end{tabular}

Table 1. Table of symbols and their definitions 